hep-ph/9605433

revised version (November 1996)

\title{
Regularization at the next-to-leading order in the top-mode standard model without gauge bosons
}

\author{
G. Cvetǐ̌ \\ Inst. für Physik, Universität Dortmund, 44221 Dortmund, Germany
}

\begin{abstract}
We study Higgs condensation $\mathcal{H} \sim \bar{t} t$ in the top-mode standard model at the next-to-leading (NTL) order in $1 / N_{\mathrm{c}}$, by calculating the effective potential as a function of a hard mass term $\sigma_{0}$ of the top quark. We include the effects of the third generation quarks, the Higgs and the Goldstone fields, and the leading QCD effects, but not the effects of the transverse components of the electroweak gauge bosons. The resulting effective theory contains finite energy cutoff parameters $\left(\Lambda_{\mathrm{f}}, \Lambda_{\mathrm{b}}\right)$ for the fermionic and the bosonic degrees of freedom. Condensation is supposed to take place at energies $\Lambda \sim \Lambda_{\mathrm{f}} \sim \Lambda_{\mathrm{b}}$. The paper describes how to regularize the integrals over the fermionic momenta in a way free of momentum branching ambiguities and how to treat the terms of $1 / N_{\mathrm{c}}$ expansion mutually consistently. This is achieved by the proper time approach, employing specifically the proper time cutoff (PTC) or a Pauli-Villars (PV) regulator. For comparison, we use also the covariant spherical (S) cutoff. On the other hand, for the bosonic momenta we have to use the covariant spherical cutoff. We discuss how to ensure the validity of Goldstone theorem. Demanding that the NTL contributions not "wash out" the leading ones, we obtain rather low bounds for the cutoffs: $\Lambda=\mathcal{O}(1 \mathrm{TeV})$. The results for the corresponding cases with PTC, PV and S regularization differ only marginally. Thus, in the described framework, $1 / N_{\mathrm{c}}$ expansion approach has a predictive power only if $\Lambda=\mathcal{O}(1 \mathrm{TeV})$, a result largely independent of the regularization procedure.
\end{abstract}




\section{Introduction}

Higgs meson could be a bound state of heavy quark pairs [1]- [16] (and references therein). This idea originates from an earlier work of Nambu and Jona-Lasinio (NJL) [17 where it was applied to low energy QCD, and independently from Vaks and Larkin [18]. The bound states (condensates) are treated in these works either in the leading- $N_{\mathrm{c}}$ approximation, or in a form that takes into account part of the effects beyond the leading- $N_{\mathrm{c}}$ - by using improved Schwinger-Dyson equations, or renormalization group equations (RGEs). A particularly straightforward NJL-type framework, containing the essential features of the idea of $\bar{t}$ condensation, is the top-mode standard model (TSM) Lagrangian, in its simplified form known also as the BHL (Bardeen-Hill-Lindner) Lagrangian [⿰]𠃌灬].

In recent works, we studied the next-to-leading order (NTL) contributions in $1 / N_{\mathrm{c}}$ expansion in TSM by including quadratic fluctuations of the composite Higgs $\mathcal{H} \sim \bar{t} t$ [19], as well as those of the

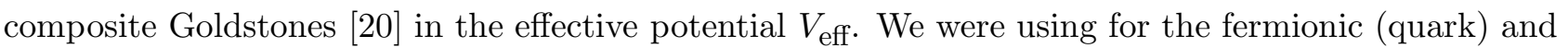
the bosonic momenta the simple covariant spherical cutoffs $\Lambda_{\mathrm{f}}, \Lambda_{\mathrm{b}}$. These two cutoffs are indicative of the energy at which the condensation is supposed to occur: $\Lambda \sim \Lambda_{\mathrm{f}} \sim \Lambda_{\mathrm{b}}$. QCD effects were included, but their impact was found to be small. We considered the effective potential as a function of a hard bare mass term $\sigma_{0}$ of the top quark, i.e., the expectation value $\sigma_{0}$ of a composite (initially auxiliary) Higgs field $\tilde{\sigma} \propto \mathcal{H}$. We demanded that the NTL contributions not "wash out" the leading- $N_{\mathrm{c}}$ ones, or equivalently, that the $1 / N_{\mathrm{c}}$ expansion approach in this framework have predictive power. As a direct consequence of this demand, we obtained that the energy at which the $\bar{t} t$ pair is supposed to condense into a Higgs is rather low: $\Lambda=\mathcal{O}(1 \mathrm{TeV})$.

In the present work, we continue the work of Refs. [19]-20]. We concentrate on various regularization procedures and the dependence of the mentioned results on these procedures. The reasons for this are at least two:

Firstly, objections may be raised against the results of [19], [20], on the grounds that they may be largely the consequence of our choosing only one specific regularization - the simple covariant spherical cutoff, and that other regularizations may give substantially different results.

Secondly, theoretical objections had been raised in the past against using simple covariant spherical cutoffs for the quark momenta [21], particularly because of the momentum branching ambiguity which has its origin in the translational noninvariance of the procedure.

One possibility to remedy this problem is to use dispersion relations (DR) with finite cutoff for

the bubble-chain-corrected scalar propagators - this method was applied by the authors of Refs. [17] 
and 22] at the leading- $N_{\mathrm{c}}$ order. The leading- $N_{\mathrm{c}}$ gap equation, determining the relationship between the four-fermion coupling, the dynamical quark mass and the cutoff is then obtained by requiring that the pole of the Goldstone propagator be located at the zero momentum. However, when we want to include the NTL corrections to the gap equation with the method of the effective potential, it is unclear how to relate the DR cutoff appearing in the NTL part $V^{(1)}$ of the effective potential (containing as integrands the logarithms of the bubble-chain-corrected scalar propagators) and the cutoff in the leading- $N_{\mathrm{c}}$ part $V^{(0)}$. For the latter part, there seems to be no dispersion relation representation available, because the integrand there contains only one (quark) momentum.

Therefore, we apply in this paper the so-called proper time regularization approach for the fermionic momenta, using the Schwinger representation for the "bosonized" effective action. This regularization, in contrast to the spherical cutoff, does not suffer from the momentum branching ambiguity problems, since it is translationally invariant in the momentum space. Furthermore, in contrast to the DR cutoff regularization, the present procedure treats mutually consistently the leading- $N_{\mathrm{c}}$ and the NTL contributions, due to the introduction of a fermion momentum regulator function that is common to both contributions.

In Sec. II, we calculate the effective potential, including the NTL contributions. We take into account the effects of the third generation quarks, and at the NTL level in addition the effects of the Higgs and the three Goldstones. The latter degrees of freedom represent, in the Landau gauge, the longitudinal degrees of freedom of the $Z$ and $W^{ \pm}$. The effects of the transverse components of the electroweak gauge bosons are not included. Several technical details of the derivation, in the proper time regularization framework, are given in Appendices A and B. We employ two different fermionic regulators in the proper time regularization framework: the proper time cutoff (PTC) regulator, and the Pauli-Villars (PV) two-subtractions regulator; for additional comparison, we also employ the simple covariant spherical cutoff (S) as used in Ref. [20]. For the bosonic momenta, which appear only at the NTL level and possess no momentum branching ambiguity in our problem, the proper time approach turns out not to lead to a regularization, and therefore we always use the covariant spherical cutoff there.

In Sec. III, we derive the NTL gap equation, i.e., the requirement of the minimum of the effective potential in the vacuum. The solutions of the NTL gap equation give us the ratio $\left(m_{t}(\Lambda) / \Lambda_{\mathrm{f}}\right)$ of the bare mass of the top quark and the fermionic cutoff parameter. Furthermore, we discuss in detail how to eliminate the singularities in the NTL integrals over the bosonic momenta in all (PTC, PV, S) cases, thus ensuring that Goldstone theorem is respected at the NTL level. 
In Sec. IV, we include the mass renormalization effects. We also include the leading QCD effects - in the gap equation and in the mass renormalization.

In Sec. V, we investigate numerically the gap equation solutions $\left.\left(\mapsto m_{t}(\Lambda) / \Lambda_{\mathrm{f}}\right)\right)$ and their mass renormalization $\left(\mapsto m_{t}^{\text {ren. }} / \Lambda_{\mathrm{f}}\right)$. The obtained values of the latter ratios lead to the values of the cutoff parameters $\Lambda_{\mathrm{f}}$ and $\Lambda_{\mathrm{b}}$ (using: $m_{t}^{\text {ren. }} \approx 180 \mathrm{GeV}$ ). We also compare in Sec. $\mathrm{V}$ how the choice of regularization of the fermionic momenta (PTC, PV, S) influences the numerical results, in particular the NTL corrections to the solutions of the gap equation. It turns out that these corrections are in the S regularization cases somewhat weaker and in the PV cases somewhat stronger than in the corresponding PTC cases. We demand that the leading- $N_{\mathrm{c}}$ and the NTL solutions of the gap equation not differ drastically, i.e., that the NTL corrections not "wash out" the leading- $N_{\mathrm{c}}$ solutions. As a consequence, it turns out that the cutoff parameters are rather low: $\Lambda_{\mathrm{f}}, \Lambda_{\mathrm{b}} \leq \mathcal{O}(1 \mathrm{TeV})$.

In Sec. VI, we recapitulate the basic conclusions of the paper and compare it with related works of other authors and, in particular, with the works [2]-[5].

\section{The model, the effective potential, proper time regularization}

The top-mode standard model (TSM) Lagrangian [4], known also as the BHL (Bardeen-Hill-Lindner) Lagrangian, contains a truncated $S U(2)_{L} \times U(1)_{Y}$ invariant four-fermion interaction at a high energy scale $E \sim \Lambda$. This term is assumed to be responsible for the creation of a composite Higgs field $\mathcal{H} \sim \bar{t} t$

$$
\mathcal{L}^{(\Lambda)}=\mathcal{L}_{\text {kin }}^{0}+G\left(\bar{\Psi}_{\mathrm{L}}^{i a} t_{\mathrm{R} a}\right)\left(\bar{t}_{\mathrm{R}}^{b} \Psi_{\mathrm{L} b}^{i}\right) \quad \text { for } E \sim \Lambda
$$

In this expression, $a$ and $b$ are the color and $i$ the isospin indices, $\Psi_{\mathrm{L}}^{T}=\left(t_{\mathrm{L}}, b_{\mathrm{L}}\right) . \mathcal{L}_{\text {kin }}^{0}$ contains the

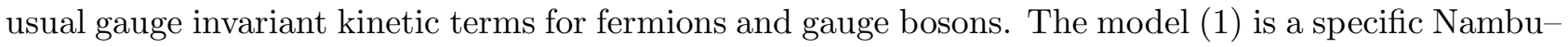
Jona-Lasinio (NJL) type model with the Standard Model symmetry $S U(2)_{L} \times U(1)_{Y}$. It leads to an effective framework for the minimal Standard Model. It can be rewritten in terms of an additional, as yet auxiliary, scalar $S U(2)_{L}$ isodoublet $\Phi$, by adding to it the following quadratic term

$$
\begin{gathered}
\mathcal{L}_{\text {new }}^{(\Lambda)}=\mathcal{L}_{\text {old }}^{(\Lambda)}-\left[M_{0} \tilde{\Phi}^{i \dagger}+\sqrt{G} \bar{\Psi}_{\mathrm{L}}^{i a} t_{\mathrm{R} a}\right]\left[M_{0} \tilde{\Phi}^{i}+\sqrt{G} \bar{t}_{\mathrm{R}}^{b} \Psi_{\mathrm{L} b}^{i}\right] \\
\text { where: } \quad \tilde{\Phi}=i \tau_{2} \Phi^{*}, \quad \Phi=\frac{1}{\sqrt{2}}\left(\begin{array}{c}
\sqrt{2} \mathcal{G}^{(+)} \\
\mathcal{H}+i \mathcal{G}^{(0)}
\end{array}\right), \quad \mathcal{G}^{( \pm)}=\frac{1}{\sqrt{2}}\left(\mathcal{G}^{(1)} \pm i \mathcal{G}^{(2)}\right) .
\end{gathered}
$$

The addition of such a term changes the generating functional only by a source independent factor [23]. Here, $\mathcal{H}, \mathcal{G}^{(0)}, \mathcal{G}^{(1)}$ and $\mathcal{G}^{(2)}$ are the Higgs and the three real Goldstone components of 
the auxiliary complex isodoublet field $\Phi$, and $M_{0}$ is an unspecified bare mass term for $\Phi$ at $E \sim$ $\Lambda$. The physical results will turn out to be independent of the specific value of $M_{0}$. These scalar fields eventually become the physical Higgs and the "scalar" longitudinal components of the massive electroweak bosons through quantum effects. We ignore the transverse components of $W^{ \pm}$and $Z$, and all the lighter quarks which we assume to be and remain massless. It can be shown that the massless Goldstones discussed here correspond to the Goldstone degrees of freedom of $W^{ \pm}$and $Z$ in the Landau gauge $(\xi \rightarrow \infty)$. In this gauge, the ghosts do not couple to the scalar degrees of freedom and therefore the ghosts do not contribute to the effective potential [24], at least not at the leading- $N_{\mathrm{c}}$ and the NTL level.

The resulting effective Lagrangian now reads

$$
\mathcal{L}^{(\Lambda)}=-\bar{\Psi}^{a} \hat{\mathcal{D}} \Psi_{a}-M_{0}^{2} \Phi^{\dagger} \Phi
$$

where

$$
\hat{\mathcal{D}}=-\hat{P}+\left[\begin{array}{c}
\left(\tilde{\sigma}_{0}-i \gamma_{5} \tilde{\sigma}_{1}\right) \\
{\left[-\frac{1}{2}\left(1+\gamma_{5}\right)\left(\tilde{\sigma}_{2}-i \tilde{\sigma}_{3}\right)\right]}
\end{array}\right]
$$

We used for the auxiliary scalar fields the following notation:

$$
\tilde{\sigma}_{0}=\sqrt{\frac{g}{2}} \mathcal{H}, \quad \tilde{\sigma}_{j}=\sqrt{\frac{g}{2}} \mathcal{G}^{(j-1)} \quad(j=1,2,3),
$$

where we introduced a dimensionless coupling constant $g=G M_{0}^{2}$. Equations of motion give

$$
\tilde{\sigma}_{0}=-\frac{G}{2}\left(\bar{t}^{a} t_{a}\right), \quad \tilde{\sigma}_{1}=i \frac{G}{2}\left(\bar{t}^{a} \gamma_{5} t_{a}\right), \quad \tilde{\sigma}^{(-)}\left(=\frac{1}{\sqrt{2}}\left(\tilde{\sigma}_{2}-i \tilde{\sigma}_{3}\right)\right)=\frac{G}{\sqrt{2}}\left[\bar{t}^{a}\left(\frac{1-\gamma_{5}}{2}\right) b_{a}\right]
$$

thus signaling that the introduced auxiliary fields, once they become physical (dynamical) through quantum effects, will represent the composite Higgs and the composite Goldstone (i.e., the composite longitudinal $Z$ and $W$ ) degrees of freedom.

The effective potential $V_{\text {eff }}\left(\Phi_{0}\right)$, as a function of the expectation values $\Phi_{0}$ of the scalar fields, can then be calculated in the Wick rotated Euclidean space by means of the following general formula

$$
\begin{gathered}
\exp \left[-\Omega V_{\text {eff }}\left(\mathcal{H}_{0}, \mathcal{G}_{0}^{(j)}\right)\right]=\text { const. } \times \int \prod_{j=0}^{2}\left[\mathcal{D} \mathcal{G}^{(j)} \delta\left(\int d^{4} \bar{y} \mathcal{G}^{(j)}(\bar{y})-\Omega \mathcal{G}_{0}^{(j)}\right)\right] \times \\
\int \mathcal{D} \mathcal{H} \delta\left(\int d^{4} \bar{y} \mathcal{H}(\bar{y})-\Omega \mathcal{H}_{0}\right) \int \mathcal{D} \bar{\Psi} \mathcal{D} \Psi \exp \left[+\int d^{4} \bar{x} \mathcal{L}(\bar{x})\right]
\end{gathered}
$$

where we set $\hbar=1$. The bars over space-time components, derivatives and momenta from now on denote Euclidean quantities. $\Omega$ is the four-dimensional volume (formally infinite). The effective potential is the energy density of the physical ground state when the order parameters $\mathcal{H}_{0}=\langle\mathcal{H}\rangle$ 
and $\mathcal{G}_{0}^{(j)}=\left\langle\mathcal{G}^{(j)}\right\rangle=0(j=0,1,2)$ are kept fixed. In the expression (耳) we explicitly kept also the expectation values $\mathcal{G}_{0}^{(j)}$, in order to show later the explicit $S U(2)_{L} \times U(1)_{Y}$ invariance of the expression. This formula is equivalent to a conventional expression for the effective potential obtained by using Legendre transformation of the ground state energy density, as shown by Higashijima [25] 1 .

As the next step, we could simply integrate out the quark degrees of freedom, as was done in Ref. 20] (for the case of $\mathcal{G}_{0}^{(j)}=0$ ). This would then naturally lead us to the imposition of the covariant spherical cutoff on the fermionic momenta. At this stage, however, we decide to follow the proper time method which will give us the possibility of regularizing the fermionic momenta in the leading- $N_{\mathrm{c}}$ and in the NTL terms in a mutually consistent way, as mentioned earlier. First we note that the fermionic operator $\hat{\mathcal{D}}$ is not positive definite and not hermitian, while $\hat{\mathcal{D}}^{\dagger} \hat{\mathcal{D}}$ is. Furthermore, the integration over the fermionic degrees of freedom would result in no imaginary part for the effective action, i.e., the Lagrangian (without the electroweak gauge bosons) has no anomalous terms. Therefore, the following identities can be used

$$
\int \mathcal{D} \bar{\Psi} \mathcal{D} \Psi \exp \left[-\int d^{4} \bar{x} \bar{\Psi} \hat{\mathcal{D}} \Psi\right]=\exp [\operatorname{Tr} \ln \hat{\mathcal{D}}]=\operatorname{Re} \exp [\operatorname{Tr} \ln \hat{\mathcal{D}}]=\exp \left[\frac{1}{2} \operatorname{Tr} \ln \hat{\mathcal{D}}^{\dagger} \hat{\mathcal{D}}\right] .
$$

Furthermore, this "bosonized" expression can be written in the Schwinger representation, as an integral over a "proper time" $\tau$

$$
\operatorname{Tr} \ln \hat{\mathcal{D}}^{\dagger} \hat{\mathcal{D}}(\Phi)-\operatorname{Tr} \ln \hat{\mathcal{D}}^{\dagger} \hat{\mathcal{D}}(0)=-\int_{0}^{\infty} \frac{d \tau}{\tau} \rho_{\mathrm{f}}(\tau)\left\{\operatorname{Tr} \exp \left[-\tau \hat{\mathcal{D}}^{\dagger} \hat{\mathcal{D}}(\Phi)\right]-\operatorname{Tr} \exp \left[-\tau \hat{\mathcal{D}}^{\dagger} \hat{\mathcal{D}}(0)\right]\right\}
$$

The fermionic proper time regulator $\rho_{\mathrm{f}}(\tau)$ has been introduced, satisfying the conventional boundary conditions 2

$$
\rho_{\mathrm{f}}(\tau)=1 \quad \text { for: } \tau \gg 1 / \Lambda_{\mathrm{f}}^{2} ; \quad \rho_{\mathrm{f}}(\tau)=0 \quad \text { for: } \tau \ll 1 / \Lambda_{\mathrm{f}}^{2},
$$

where $\Lambda_{\mathrm{f}}$ represents an effective cutoff parameter for the fermionic momenta; it is of the same order of magnitude as the cutoff of the discussed effective theory (11)-(四): $\Lambda_{\mathrm{f}} \sim \Lambda$. After integrating out the fermionic (quark) degrees of freedom in this way, we obtain

$$
\begin{gathered}
\exp \left[-\Omega V_{\text {eff }}\left(\left\{\sigma_{j}\right\}\right)\right]=\text { const. } \times \prod_{j=0}^{3} \int_{-\infty}^{+\infty} d J_{j} \int \mathcal{D} s_{0} \mathcal{D} s_{1} \mathcal{D} s_{2} \mathcal{D} s_{3} \times \exp \left\{-\frac{1}{2} \int_{0}^{\infty} \frac{d \tau}{\tau} \rho_{\mathrm{f}}(\tau) \times\right. \\
\left.\left[\operatorname{Tr} e^{-\tau \hat{\mathcal{D}}^{\dagger} \hat{\mathcal{D}}\left(\left\{\sigma_{i}+s_{i}\right\}\right)}-\operatorname{Tr} e^{-\tau \hat{\mathcal{D}}^{\dagger} \hat{\mathcal{D}}(0)}\right]-\frac{1}{G} \int d^{4} \bar{x} \sum_{k=0}^{3}\left(\sigma_{k}+s_{k}\right)^{2}-i \sum_{k=0}^{3} J_{k} \int d^{4} \bar{x} s_{k}(\bar{x})\right\} .
\end{gathered}
$$

\footnotetext{
${ }^{1}$ Higashijima has shown the equivalence of the two approaches for the case of one scalar component (Higgs); however, the extension of his argument to several scalar components with nonzero expectation values is straightforward.

${ }^{2}$ The proper time techniques are described, for example, in a review article by R.D. Ball [26]; the Schwinger representation (9) has its origin in the mathematical identity (B.19).
} 
We denoted by $s_{k}(\bar{x})$ the rescaled quantum fluctuations of the Higgs $(k=0)$ and the Goldstones $(k=1,2,3)$

$$
\sigma_{0}+s_{0}(\bar{x})=\tilde{\sigma}_{0}(\bar{x})=\sqrt{\frac{g}{2}} \mathcal{H}(\bar{x}), \quad \sigma_{j}+s_{j}(\bar{x})=\tilde{\sigma}_{j}(\bar{x})=\sqrt{\frac{g}{2}} \mathcal{G}^{(j-1)}(\bar{x}), \quad(j=1,2,3) .
$$

Furthermore, we rewrote in (11) the $\delta$ functions of (7) as integrals over the "sources" $J_{k}$

$$
\delta\left(\int d^{4} \bar{x} s_{k}(\bar{x})\right)=\frac{1}{2 \pi} \int_{-\infty}^{+\infty} d J_{k} \exp \left[-i J_{k} \int d^{4} \bar{x} s_{k}(\bar{x})\right] .
$$

We note that in the physical vacuum we have the following expectation values:

$$
\sigma_{0}\left(=\sqrt{\frac{g}{2}}\langle\mathcal{H}\rangle\right)=m_{t}(\Lambda), \quad \sigma_{k}=0(\text { for: } k=1,2,3),
$$

where $m_{t}(\Lambda)$ is the bare mass of the top quark in the effective theory (1)-(14) after the dynamical symmetry breaking.

Now we perform the expansion of the action in the exponent of (11) in powers of the scalar quantum fluctuations $s_{k}(\bar{x})(k=0,1,2,3)$ up to and including the quadratic terms. It turns out that the terms linear in $s_{k}$, except for those proportional to $J_{k} s_{k}$ (i.e., the $\delta$ functions), do not contribute to the effective action in the exponent of (11), precisely because of the $\delta$ functions. The terms with no fluctuations then yield the leading- $N_{\mathrm{c}}$ contribution $N_{\mathrm{c}} V^{(0)}$, and the terms quadratic in fluctuations yield the next-to-leading (NTL) contribution $V^{(1)}$ of the formal $\left(1 / N_{\mathrm{c}}\right)$ expansion

$$
V_{\text {eff }}\left(\left\{\sigma_{j}\right\}\right)=N_{\mathrm{c}} V^{(0)}+V^{(1)}+\mathcal{O}\left(\frac{1}{N_{\mathrm{c}}}\right) .
$$

The leading- $N_{\mathrm{c}}$ contribution can therefore be read off immediately from (11)

$$
N_{\mathrm{c}} V^{(0)}\left(\left\{\sigma_{j}\right\}\right)=\frac{1}{G} \sum_{j=0}^{3} \sigma_{j}^{2}+\frac{1}{2 \Omega} \int_{0}^{\infty} \frac{d \tau}{\tau} \rho_{\mathrm{f}}(\tau)\left[\operatorname{Tr} e^{-\tau \hat{\mathcal{D}}^{\dagger} \hat{\mathcal{D}}\left(\left\{\sigma_{i}\right\}\right)}-\operatorname{Tr} e^{-\tau \hat{\mathcal{D}}^{\dagger} \hat{\mathcal{D}}(0)}\right] .
$$

In Appendix A we wrote down the entire expression for the hermitean operator matrix $\hat{\mathcal{D}}^{\dagger} \hat{\mathcal{D}}\left(\left\{\sigma_{j}\right\}\right)$ in the Euclidean metric. The matrix has dimension $8 \times 8$ in the combined spinor and isospin space [cf.(14)], and we diagonalized it there by means of a unitary matrix $U$

$$
U^{\dagger} \hat{\mathcal{D}}^{\dagger} \hat{\mathcal{D}}\left(\left\{\sigma_{j}\right\}\right) U=\hat{\bar{P}} \cdot \hat{\bar{P}}+\sum_{j=0}^{3} \sigma_{j}^{2}\left[\begin{array}{ll}
1 & 0 \\
0 & 0
\end{array}\right]
$$

The matrix above is a block matrix made up of blocks of dimension $4 \times 4$. From here we see that only the top quark isospin component contributes to $N_{\mathrm{c}} V^{(0)}$ in the physical vacuum, since the off-diagonal elements in $U$ matrix of (A.4) are zero then: $g_{0}^{( \pm)}=0$. Inserting (17) into (16), performing the tracing 
over the coordinates in the momentum basis, and tracing also in the color and isospin space, we end up with the general leading- $N_{\mathrm{c}}$ contribution in the proper time approach

$$
\begin{aligned}
N_{\mathrm{c}} V^{(0)}\left(\Phi_{0}^{\dagger} \Phi_{0}\right) & =M_{0}^{2} \Phi_{0}^{\dagger} \Phi_{0}+\frac{1}{2 \Omega} \int_{0}^{\infty} \frac{d \tau}{\tau} \rho_{\mathrm{f}}(\tau) N_{c} 4 \Omega \int \frac{d^{4} \bar{q}}{(2 \pi)^{4}}\left\{\exp \left[-\tau\left(\bar{q}^{2}+g \Phi_{0}^{\dagger} \Phi_{0}\right)\right]-\exp \left[-\tau \bar{q}^{2}\right]\right\} \\
& =M_{0}^{2} \Phi_{0}^{\dagger} \Phi_{0}+\frac{N_{\mathrm{c}}}{8 \pi^{2}} \int_{0}^{\infty} \frac{d \tau}{\tau^{3}} \rho_{\mathrm{f}}(\tau)\left[\exp \left(-\tau g \Phi_{0}^{\dagger} \Phi_{0}\right)-1\right]
\end{aligned}
$$

where the factors $N_{\mathrm{c}}$ and 4 come from tracing the identity matrix in the color space and in the spinor space of the top quark, respectively. We denoted by $\Phi_{0}$ simply the expectation value of the scalar isodoublet, i.e.,

$$
\Phi_{0}^{\dagger} \Phi_{0}=\frac{1}{2}\left[\mathcal{H}_{0}^{2}+\sum_{k=0}^{2} \mathcal{G}_{0}^{(k) 2}\right]=\frac{1}{g} \sum_{j=0}^{3} \sigma_{j}^{2}=\frac{1}{g} \sigma^{2},
$$

and we introduced the notation $\sigma^{2}=\sum_{0}^{3} \sigma_{j}^{2}$. From (18) we see explicitly that the leading- $N_{\mathrm{c}}$ contribution is a function of the $S U(2)_{L} \times U(1)_{Y}$ invariant $\Phi_{0}^{\dagger} \Phi_{0}$, and is therefore itself an $S U(2)_{L} \times U(1)_{Y}$ invariant expression, as it should be. Certainly, in our calculations we will take at the end $\Phi_{0}^{\dagger} \Phi_{0}=\sigma_{0}^{2} / g=m_{t}^{2}(\Lambda) / g$, since we will be searching for the physical vacuum that dynamically breaks the $S U(2)_{L} \times U(1)_{Y}$ to $U(1)_{\mathrm{em}}$.

The leading- $N_{\mathrm{c}}$ expression (18) can be obtained also diagrammatically, in its nonregularized form, by calculating the 1-PI one-loop Green functions corresponding to the diagrams of Fig. 1 with outer legs of zero momentum. This is described in detail in [27].

At this point, we introduce two specific choices for the fermionic (quark) proper time regulator $\rho_{\mathrm{f}}(\tau)$ (i.e., the regularization for the fermionic momenta of the model): proper time cutoff (PTC), and the Pauli-Villars (PV) regularization with two subtractions:

$$
\begin{aligned}
& \rho_{\mathrm{f}}^{(\mathrm{PTC})}(\tau)=\Theta\left(\tau-1 / \Lambda_{\mathrm{f}}^{2}\right)=\left\{\begin{array}{cc}
1 & \text { for: } \tau>1 / \Lambda_{\mathrm{f}}^{2} \\
0 & \text { for: } \tau<1 / \Lambda_{\mathrm{f}}^{2}
\end{array}\right\}, \\
& \rho_{\mathrm{f}}^{(\mathrm{PV})}(\tau)=\left[1-\exp \left(-\tau \Lambda_{\mathrm{f}}^{2}\right)\right]^{2}=1-2 e^{-\tau \Lambda_{\mathrm{f}}^{2}}+e^{-\tau 2 \Lambda_{\mathrm{f}}^{2}} .
\end{aligned}
$$

The leading- $N_{\mathrm{c}}$ part $N_{\mathrm{c}} V^{(0)}\left(\sigma^{2}\right)$ of the effective potential was calculated explicitly for these two cases (PTC, PV) in Appendix B. We note that the two $\Lambda_{\mathrm{f}}$ 's in (20) and (21) are not equal in the corresponding cases with equal four-fermion coupling $G=8 \pi^{2} a /\left(N_{\mathrm{c}} \Lambda_{\mathrm{f}}^{2}\right)$ of the Lagrangian (1). In fact, if we require that $\Lambda_{\mathrm{f}}^{2}$ terms in $N_{\mathrm{c}} \partial V^{(0)} / \partial \sigma^{2}$ be equal in the two cases, we obtain [cf.(B.4)-(B.5)]: $\Lambda_{\mathrm{f}}^{2}(\mathrm{PTC})=(2 \ln 2) \Lambda_{\mathrm{f}}^{2}(\mathrm{PV})$. The $\ln \Lambda_{\mathrm{f}}^{2}$ terms then agree automatically with each other. 
Next we turn to the derivation of the next-to-leading (NTL) contribution $V^{(2)}$. First we expand $\hat{\mathcal{D}}^{\dagger} \hat{\mathcal{D}}$ up to quadratic terms in the scalar fluctuations $\left\{s_{j}(\bar{x})\right\}$ (12)

$$
\hat{\mathcal{D}}^{\dagger} \hat{\mathcal{D}}\left(\left\{\sigma_{j}+s_{j}(\bar{x})\right\}\right)=\hat{\triangle}_{0}\left(\left\{\sigma_{j}\right\}\right)+\hat{\triangle}_{1}\left(\left\{\sigma_{j} ; s_{j}(\bar{x})\right\}\right)+\hat{\triangle}_{2}\left(\left\{\sigma_{j} ; s_{j}(\bar{x})\right\}\right)+\cdots
$$

Operators $\hat{\triangle}_{1}$ and $\hat{\triangle}_{2}$ are linear and quadratic in the scalar fluctuations $\left\{s_{j}(\bar{x})\right\}$, and the dots denote terms which are at least cubic in $\left\{s_{j}(\bar{x})\right\}$. Explicit expressions for $\hat{\triangle}_{1}$ and $\hat{\triangle}_{2}$ are given in (B.6)-(B.8) 5 . Furthermore, we denoted: $\hat{\triangle}_{0}=\hat{\mathcal{D}}^{\dagger} \hat{\mathcal{D}}\left(\left\{\sigma_{j}\right\}\right)$. Inserting expansion (22) into (11), we then obtain the NTL contribution $V^{(1)}$ to the effective potential by keeping only the contributions quadratic in the fluctuations (and by keeping the $\delta$ functions, as mentioned earlier)

$$
\begin{aligned}
\exp [- & \left.\Omega V^{(1)}\left(\left\{\sigma_{j}\right\}\right)\right]=\text { const. } \times \prod_{j=0}^{3} \int_{-\infty}^{+\infty} d J_{j} \int \mathcal{D} s_{0} \mathcal{D} s_{1} \mathcal{D} s_{2} \mathcal{D} s_{3} \times \\
\exp & \left\{+\frac{1}{2} \int_{0}^{\infty} d \tau \rho_{\mathrm{f}}(\tau) \operatorname{Tr}\left[e^{-\tau \hat{\triangle}_{0}\left(\left\{\sigma_{k}\right\}\right)} \hat{\triangle}_{2}\left(\left\{\sigma_{k} ; s_{k}\right\}\right)\right]\right. \\
& -\frac{1}{4} \int_{0}^{\infty} d \tau_{1} \int_{0}^{\infty} d \tau_{2} \rho_{\mathrm{f}}\left(\tau_{1}+\tau_{2}\right) \operatorname{Tr}\left[e^{-\tau_{1} \hat{\triangle}_{0}\left(\left\{\sigma_{k}\right\}\right)} \hat{\triangle}_{1}\left(\left\{\sigma_{k} ; s_{k}\right\}\right) e^{-\tau_{2} \hat{\triangle}_{0}\left(\left\{\sigma_{k}\right\}\right)} \hat{\triangle}_{1}\left(\left\{\sigma_{k} ; s_{k}\right\}\right)\right] \\
& \left.-\frac{1}{G} \int d^{4} \bar{x} \sum_{k=0}^{3} s_{k}(\bar{x})^{2}-i \sum_{k=0}^{3} J_{k} \int d^{4} \bar{x} s_{k}(\bar{x})\right\} .
\end{aligned}
$$

We calculate the above traces in the momentum basis and in the unitarily rotated spinor-isospin basis in which the $8 \times 8$ matrix $\hat{\triangle}_{0}\left(\left\{\sigma_{j}\right\}\right)=\hat{\mathcal{D}}^{\dagger} \hat{\mathcal{D}}\left(\left\{\sigma_{j}\right\}\right)$ is diagonal [cf.(17)]. The steps and some details are explained in Appendix B. It turns out that the proper time action in the curly brackets of the exponent in (23) can be written in the following form:

$$
-\frac{1}{2} \iint d^{4} \bar{x} d^{4} \bar{y} v_{j}(\bar{y}) \hat{\mathcal{A}}_{j}\left(\bar{y}, \bar{x} ; \sigma^{2}\right) v_{j}(\bar{x})-i I_{j} \int d^{4} \bar{x} v_{j}(\bar{x}),
$$

where the summation over all $j=0, \ldots, 3$ is implied, the fluctuations $v_{j}(\bar{x})$ are obtained from the original scalar fluctuations $s_{j}(\bar{x})$ by an orthonormal transformation $\mathcal{O}$ given in $(\mathrm{B} .12)-(\mathrm{B} .13)$, and the "sources" $I_{j}$ 's are obtained from $J_{j}$ 's by the same transformation. Matrix $\mathcal{O}$ is constructed in such a way that the action terms quadratic in fluctuations are now diagonal. Furthermore, the diagonal kernel elements $\hat{\mathcal{A}}_{j}\left(\bar{y}, \bar{x} ; \sigma^{2}\right)$ depend on the $S U(2)_{L} \times U(1)_{Y}$ invariant expectation value $\sigma^{2}=\sum_{0}^{3} \sigma_{j}^{2}=g \Phi_{0}^{\dagger} \Phi_{0}$ and are translationally invariant in the (Euclidean) configuration space, i.e., they are functions of the difference $\bar{x}-\bar{y}$. They are obtained explicitly in Appendix B. Since the Jacobian of any orthonormal transformation is equal to one, we can replace in the path integral (23) the integrations over the

\footnotetext{
${ }^{3}$ Expressions in (22) are local functions in the $\bar{x}$ space. Formally, this should be understood in the $|\bar{x}\rangle$ basis as: $\left\langle\bar{x}\left|\hat{\mathcal{D}}^{\dagger} \hat{\mathcal{D}}\left(\left\langle\sigma_{j}+s_{j}\right\rangle\right)\right| \bar{x}^{\prime}\right\rangle=\delta^{(4)}\left(\bar{x}-\bar{x}^{\prime}\right) \hat{\mathcal{D}}^{\dagger} \hat{\mathcal{D}}\left(\left\langle\sigma_{j}+s(\bar{x})_{j}\right\rangle\right)$.
} 
fluctuations $s_{j}$ and sources $J_{j}$ by integrations over $v_{j}$ and $I_{j}$, respectively. Therefore, we end up with the diagonal integrals of the Gaussian type which can be solved

$$
\begin{aligned}
& \int \mathcal{D} v_{j} \int_{-\infty}^{+\infty} d I_{j} \exp \left\{-\frac{1}{2} \iint d^{4} \bar{x} d^{4} \bar{y} v_{j}(\bar{y}) \hat{\mathcal{A}}_{j}\left(\bar{y}, \bar{x} ; \sigma^{2}\right) v_{j}(\bar{x})-i I_{j} \int d^{4} \bar{x} v_{j}(\bar{x})\right\} \\
& =\exp \left[-\frac{1}{2} \operatorname{Tr} \ln \hat{\mathcal{A}}_{j}\left(\sigma^{2}\right)\right] \int_{-\infty}^{+\infty} d I_{j} \exp \left[-\frac{1}{2} I_{j}^{2} \iint d^{4} \bar{x} d^{4} \bar{y} \hat{\mathcal{A}}_{j}^{-1}\left(\bar{y}, \bar{x} ; \sigma^{2}\right)\right] \\
& =\exp \left[-\frac{1}{2} \operatorname{Tr} \ln \hat{\mathcal{A}}_{j}\left(\sigma^{2}\right)\right] \sqrt{\frac{2 \pi}{\Omega}} \sqrt{\tilde{A}_{j}\left(\bar{p}^{2}=0 ; \sigma^{2}\right)}
\end{aligned}
$$

where we denoted by $\tilde{A}$ the Fourier transform of $\hat{\mathcal{A}}_{j}\left(\bar{y}, \bar{x} ; \sigma^{2}\right)$

$$
\tilde{A}_{j}\left(\bar{p}^{2} ; \sigma^{2}\right)=\int d^{4} \bar{x} \exp [-i \bar{p} \cdot \bar{x}] \hat{\mathcal{A}}_{j}\left(0, \bar{x} ; \sigma^{2}\right) .
$$

Taking the logarithm, we end up with the following expression for the NTL part $V^{(1)}$ of the effective potential

$$
V^{(1)}\left(\sigma^{2}\right)=\frac{1}{2 \Omega} \sum_{j=0}^{3} \operatorname{Tr} \ln \hat{\mathcal{A}}_{j}\left(\sigma^{2}\right)-\frac{1}{2 \Omega} \sum_{j=0}^{3} \ln \tilde{A}\left(\bar{p}^{2}=0 ; \sigma^{2}\right) .
$$

The second sum on the RHS of (27), which is the remnant of the $\delta$ function conditions, is evidently zero in the infinite volume limit $\left(\Omega=\int d^{4} \bar{x} \rightarrow \infty\right)$. On the other hand, the first sum is finite. Performing the tracing in the (bosonic) momentum basis, we obtain finally

$$
V^{(1)}\left(\sigma^{2}\right)=\frac{1}{32 \pi^{2}} \sum_{j=0}^{3} \int_{0}^{\Lambda_{\mathrm{b}}^{2}} d \bar{p}^{2} \bar{p}^{2} \ln \tilde{A}_{j}\left(\bar{p}^{2} ; \sigma^{2}\right) .
$$

Here we introduced a finite spherical cutoff $\Lambda_{\mathrm{b}}\left(\Lambda_{\mathrm{b}} \sim \Lambda_{\mathrm{f}} \sim \Lambda\right)$ for the bosonic momenta $\bar{p}$. If we hadn't cut off the integration this way, and if we had tried to regularize the bosonic momenta again with the proper time method, we would have ended up with severely divergent integrals over the bosonic proper times, since the effective actions $\tilde{A}_{j}$ in momentum space are not proportional to $\bar{p}^{2}$ as $\bar{p}^{2} \rightarrow \infty$ (i.e., no kinetic terms for bosons at $\bar{p}^{2} \gg \Lambda_{\mathrm{f}}^{2}$ ), but rather converge to a constant. However, the spherical cutoff for the bosonic momenta is not as problematic as it is for the fermionic momenta. Namely, unlike the fermionic spherical cutoff case, the integrals over the bosonic momenta evidently don't suffer in our expressions from the momentum branching ambiguities - the integrands in (28) don't depend on scalar products $\bar{p} \cdot \bar{k}\left(\bar{k}\right.$ being a fermionic momentum), but only on $\bar{p}^{2}$.

The kernels $\hat{\mathcal{A}}_{j}$ have the following structure in the $\bar{x}$ basis:

$$
\begin{aligned}
{\left[\begin{array}{l}
\hat{\mathcal{A}}_{0}\left(0, \bar{x} ; \sigma^{2}\right) \\
\hat{\mathcal{A}}_{1}\left(0, \bar{x} ; \sigma^{2}\right)
\end{array}\right] } & =\alpha^{(1)}\left(\sigma^{2}\right) \delta(\bar{x})+\left(\beta_{1}^{(1)} \pm \beta_{2}^{(1)}\right)\left(\bar{x} ; \sigma^{2}\right), \\
\hat{\mathcal{A}}_{2}\left(0, \bar{x} ; \sigma^{2}\right) & =\left[\alpha^{(1)}\left(\sigma^{2}\right)+\alpha^{(2)}\left(\sigma^{2}\right)\right] \delta(\bar{x})+\beta^{(2)}\left(\bar{x} ; \sigma^{2}\right)=\hat{\mathcal{A}}_{3}\left(0, \bar{x} ; \sigma^{2}\right),
\end{aligned}
$$


where the explicit expressions for the functions $\alpha^{(k)}$ and $\beta^{(k)}$ in terms of the integrals over the proper time are given in (B.10)-(B.11). In the momentum basis, applying (28), we finally get for the NTL part of the effective potential

$$
\begin{aligned}
& V^{(1)}\left(\sigma^{2}\right)=\frac{1}{32 \pi^{2}} \int_{0}^{\Lambda_{\mathrm{b}}} d \bar{p}^{2} \bar{p}^{2}\left\{\quad+\ln \left[\alpha^{(1)}\left(\sigma^{2}\right)+\left(\tilde{\beta}_{1}^{(1)}+\tilde{\beta}_{2}^{(1)}\right)\left(\bar{p}^{2} ; \sigma^{2}\right)\right]\right. \\
& +\ln \left[\alpha^{(1)}\left(\sigma^{2}\right)+\left(\tilde{\beta}_{1}^{(1)}-\tilde{\beta}_{2}^{(1)}\right)\left(\bar{p}^{2} ; \sigma^{2}\right)\right] \\
& \left.+2 \ln \left[\alpha^{(1)}\left(\sigma^{2}\right)+\alpha^{(2)}\left(\sigma^{2}\right)+\tilde{\beta}^{(2)}\left(\bar{p}^{2} ; \sigma^{2}\right)\right]\right\},
\end{aligned}
$$

where $\tilde{\beta}_{j}^{(k)}$ 's are the Fourier transforms of $\beta_{j}^{(k)}$ 's. Note that the three terms on the RHS of (30) correspond to the NTL contributions of the Higgs, the neutral Goldstone and the two charged Goldstones, respectively. From now on, we will take the $S U(2)_{L} \times U(1)_{Y}$ invariant square of the field expectation value $g \Phi_{0}^{\dagger} \Phi_{0}=\sigma^{2}=\sum_{0}^{3} \sigma_{j}^{2}$ in these formulas to be equal to the square of the (rescaled) Higgs expectation value $\sigma_{0}^{2}=g \mathcal{H}_{0}^{2} / 2$, i.e., we will search for the physical vacuum.

It turns out, as shown in Appendix B, that $\alpha^{(1)}\left(\sigma_{0}^{2}\right)$ is in general exactly twice the derivative of the leading- $N_{\mathrm{c}}$ part of the effective potential

$$
\alpha^{(1)}\left(\sigma_{0}^{2}\right)=2 \frac{\partial}{\partial \sigma_{0}^{2}}\left[N_{\mathrm{c}} V^{(0)}\left(\sigma_{0}^{2}\right)\right] .
$$

This quantity is zero by definition if $\sigma_{0}$ is the solution $\sigma_{0}=m_{t}^{(0)}$ of the leading- $N_{\mathrm{c}}$ gap equation. However, if $\sigma_{0}$ is equal (or in the vicinity) of the NTL gap equation solution, i.e., if $\sigma_{0}^{2}=m_{t}^{(0) 2}(1+$ $\left.\mathcal{O}\left(1 / N_{\mathrm{C}}\right)\right)$, then $\alpha^{(1)}\left(\sigma_{0}^{2}\right)$ is formally of order $\mathcal{O}\left(N_{\mathrm{c}} *\left(1 / N_{\mathrm{c}}\right)\right)=\mathcal{O}\left(N_{\mathrm{c}}^{0}\right)$ in the $\left(1 / N_{\mathrm{c}}\right)$ expansion, and it changes sign from minus to plus as $\sigma_{0}$ increases across $m_{t}^{(0)}$. On the other hand, the rest of the arguments in each of the logarithms in (30) is always positive and formally of order $\mathcal{O}\left(N_{\mathrm{c}}\right)$, as can be immediately seen from (B.17)-(B.18). For any specific regulator $\rho_{\mathrm{f}}(\tau)$, the explicit expressions for all these arguments can be calculated from the corresponding general formulas $(\overline{B .10})$ and $(\bar{B} .17)-(\bar{B} .18)$. Here we write down the results for the PV case (21), as an explicit series in inverse powers of the cutoff parameter $\Lambda_{\mathrm{f}}$ :

$$
\begin{aligned}
& \alpha^{(1)}\left(\sigma_{0}^{2} ; G\right)^{(\mathrm{PV})}= \frac{2}{G}-\frac{N_{\mathrm{c}}}{4 \pi^{2}}\left\{(2 \ln 2) \Lambda_{\mathrm{f}}^{2}-\sigma_{0}^{2} \ln \left(\frac{\Lambda_{\mathrm{f}}^{2}}{\sigma_{0}^{2}}\right)+\sigma_{0}^{2}(\ln 2-1)-\frac{3}{4} \frac{\sigma_{0}^{4}}{\Lambda_{\mathrm{f}}^{2}}+\cdots\right. \\
&\left.+\cdots+\frac{(-1)^{n+1}}{n(n-1)} \frac{\left(2^{n}-1\right)}{2^{n-1}} \frac{\sigma_{0}^{2 n}}{\Lambda_{\mathrm{f}}^{2 n-2}}+\cdots\right\} \\
&\left(\tilde{\beta}_{1}^{(1)} \pm \tilde{\beta}_{2}^{(1)}\right)\left(\bar{p}^{2} ; \sigma_{0}^{2}\right)^{(\mathrm{PV})}=\frac{N_{\mathrm{c}}}{8 \pi^{2}}\left[\bar{p}^{2}+2 \sigma_{0}^{2}(1 \pm 1)\right]\left\{\ln \left(\frac{\Lambda_{\mathrm{f}}^{2}}{2 \sigma_{0}^{2}}\right)-\left.\frac{2}{3} z F(z)\right|_{z=\bar{p}^{2} /\left(\bar{p}^{2}+4 \sigma_{0}^{2}\right)}\right.
\end{aligned}
$$




$$
\begin{gathered}
+\left[\frac{3}{2} \frac{\sigma_{0}^{2}}{\Lambda_{\mathrm{f}}^{2}}+\frac{1}{4} \frac{\bar{p}^{2}}{\Lambda_{\mathrm{f}}^{2}}\right]-\frac{7}{8}\left[\left(\frac{\sigma_{0}^{2}}{\Lambda_{\mathrm{f}}^{2}}\right)^{2}+\frac{1}{3} \frac{\sigma_{0}^{2} \bar{p}^{2}}{\Lambda_{\mathrm{f}}^{4}}+\frac{1}{30}\left(\frac{\bar{p}^{2}}{\Lambda_{\mathrm{f}}^{2}}\right)^{2}\right] \\
\left.+\sum_{n=3}^{\infty} \frac{(-1)^{n+1}}{n} \frac{\left(2^{n+1}-1\right)}{2^{n}}\left(\frac{\bar{p}^{2}}{\Lambda_{\mathrm{f}}^{2}}\right)^{n} \int_{0}^{1} d u\left[u(1-u)+\frac{\sigma_{0}^{2}}{\bar{p}^{2}}\right]^{n}\right\}, \\
{\left[\alpha^{(2)}\left(\sigma_{0}^{2}\right)+\tilde{\beta}^{(2)}\left(\bar{p}^{2} ; \sigma_{0}^{2}\right)\right]^{(\mathrm{PV})}=\frac{N_{\mathrm{c}}}{8 \pi^{2}} \bar{p}^{2}\left\{\ln \left(\frac{\Lambda_{\mathrm{f}}^{2}}{2 \sigma_{0}^{2}}\right)+\left[-\left(1+\frac{\sigma_{0}^{2}}{\bar{p}^{2}}\right)^{2} \ln \left(1+\frac{\bar{p}^{2}}{\sigma_{0}^{2}}\right)+\right.\right.} \\
\left.+\frac{\sigma_{0}^{2}}{\bar{p}^{2}}+2\right]+\left[\frac{\sigma_{0}^{2}}{\Lambda_{\mathrm{f}}^{2}}+\frac{1}{4} \frac{\bar{p}^{2}}{\Lambda_{\mathrm{f}}^{2}}\right]-\frac{7}{16}\left[\left(\frac{\sigma_{0}^{2}}{\Lambda_{\mathrm{f}}^{2}}\right)^{2}+\frac{2}{5} \frac{\sigma_{0}^{2} \bar{p}^{2}}{\Lambda_{\mathrm{f}}^{4}}+\frac{1}{15}\left(\frac{\bar{p}^{2}}{\Lambda_{\mathrm{f}}^{2}}\right)^{2}\right] \\
\left.+\sum_{n=3}^{\infty} \frac{(-1)^{n+1}}{n} \frac{\left(2^{n+1}-1\right)}{2^{n-1}}\left(\frac{\bar{p}^{2}}{\Lambda_{\mathrm{f}}^{2}}\right)^{n} \int_{0}^{1} d u u^{n+1}\left(1+\frac{\sigma_{0}^{2}}{\bar{p}^{2}}-u\right)^{n}\right\} .
\end{gathered}
$$

Function $F(z)$, appearing in $\Lambda_{\mathrm{f}}^{2}$-independent part of $\tilde{\beta}_{1}^{(1)} \pm \tilde{\beta}_{2}^{(1)}$, is given in Appendix B in (B.22).

The corresponding expressions for the PTC case are similar and were calculated explicitly in (B.14) and (B.20)-(B.21). As mentioned toward the end of Appendix B, the PV results (33)-(34) can be calculated from formulas (B.17)-(B.18) in a way very similar as in the PTC case. Moreover, we can even obtain closed analytical expressions for these quantities in the PV case [cf. (B.23)-(B.25)].

The NTL part of the effective potential, in its nonregularized form, can be calculated also diagrammatically, by evaluating the 1-PI multi-loop Green functions of the "beads" diagrams of Fig. 2. The calculation is described in detail in Appendix C of Ref. [19], and its extension to the case when Goldstone bosons are included is mentioned in [20].

\section{Gap equation at the next-to-leading order}

In order to write down the NTL gap equation that is suitable for numerical evaluations, it is useful to define the dimensionless analogues of: the momenta, the scalar expectation values, the proper time, the four-fermion coupling and the effective potential. We rescale the bosonic momenta $\bar{p}^{2} \rightarrow \Lambda_{\mathrm{f}}^{2} \bar{p}^{2}$, and introduce the following dimensionless analogues:

$$
\begin{gathered}
\varepsilon^{2}=\frac{\sigma_{0}^{2}}{\Lambda_{\mathrm{f}}^{2}}=\frac{G M_{0}^{2}}{2 \Lambda_{\mathrm{f}}^{2}}\langle\mathcal{H}\rangle^{2}, \quad a=\frac{\left(G N_{\mathrm{c}} \Lambda_{\mathrm{f}}^{2}\right)}{\left(8 \pi^{2}\right)}, \quad z=\tau \Lambda_{\mathrm{f}}^{2}, \\
\Xi_{\mathrm{eff}}=8 \pi^{2} V_{\mathrm{eff}} /\left(N_{\mathrm{c}} \Lambda_{\mathrm{f}}^{4}\right)=\Xi^{(0)}+\frac{1}{N_{\mathrm{c}}} \Xi^{(1)}+\mathcal{O}\left(\frac{1}{N_{\mathrm{c}}^{2}}\right) .
\end{gathered}
$$


The dimensionless coupling parameter $a$ is of order 1 by the leading- $N_{\mathrm{c}}$ gap equation, i.e., $a$ is formally of order $\mathcal{O}\left(N_{\mathrm{c}}^{0}\right)$ in $1 / N_{\mathrm{c}}$ expansion, as will be shown explicitly below. The resulting expressions for the leading $-N_{\mathrm{c}}$ term $\Xi^{(0)}$ and the NTL term $\Xi^{(1)}$ are

$$
\begin{gathered}
\Xi^{(0)}\left(\varepsilon^{2} ; a\right)=\frac{\varepsilon^{2}}{a}+\int_{0}^{\infty} \frac{d z}{z^{3}} \operatorname{reg}_{\mathrm{f}}(z)\left(e^{-z \varepsilon^{2}}-1\right) \\
\Xi^{(1)}\left(\varepsilon^{2} ; \Lambda_{\mathrm{b}}^{2} / \Lambda_{\mathrm{f}}^{2} ; a\right)=\frac{1}{4} \int_{0}^{\Lambda_{\mathrm{b}}^{2} / \Lambda_{\mathrm{f}}^{2}} d \bar{p}^{2} \bar{p}^{2}\left\{\ln \left[A^{(1)}\left(\varepsilon^{2} ; a\right)+\left(\tilde{B}_{1}^{(1)}+\tilde{B}_{2}^{(1)}\right)\left(\bar{p}^{2} ; \varepsilon^{2}\right)\right]\right. \\
\left.+\ln \left[A^{(1)}\left(\varepsilon^{2} ; a\right)+\left(\tilde{B}_{1}^{(1)}-\tilde{B}_{2}^{(1)}\right)\left(\bar{p}^{2} ; \varepsilon^{2}\right)\right]+2 \ln \left[A^{(1)}\left(\varepsilon^{2} ; a\right)+A^{(2)}\left(\varepsilon^{2}\right)+\tilde{B}^{(2)}\left(\bar{p}^{2} ; \varepsilon^{2}\right)\right]\right\},
\end{gathered}
$$

where $\operatorname{reg}_{\mathrm{f}}(z)=\rho_{\mathrm{f}}\left(\tau=z / \Lambda_{\mathrm{f}}^{2}\right)$, i.e., $\operatorname{reg}_{\mathrm{f}}(z)=\theta(z-1)$ in the PTC case, and $\operatorname{reg}_{\mathrm{f}}(z)=\left(1-e^{-z}\right)^{2}$ in the PV case. The dimensionless functions $A^{(j)}$ and $\tilde{B}_{k}^{(j)}$ are

$$
\begin{aligned}
A^{(j)}\left(\varepsilon^{2}\right) & =\frac{8 \pi^{2}}{N_{\mathrm{c}} \Lambda_{\mathrm{f}}^{2}} \alpha^{(j)}\left(\sigma_{0}^{2}\right), \quad A^{(1)}=2 \frac{\partial \Xi^{(0)}}{\partial \varepsilon^{2}}, \\
\tilde{B}_{k}^{(j)}\left(\bar{p}^{2} ; \varepsilon^{2}\right) & =\frac{8 \pi^{2}}{N_{\mathrm{c}} \Lambda_{\mathrm{f}}^{2}} \tilde{\beta}_{k}^{(j)}\left(\bar{q}^{2}=\Lambda_{\mathrm{f}}^{2} \bar{p}^{2} ; \sigma_{0}^{2}=\Lambda_{\mathrm{f}}^{2} \varepsilon^{2}\right) .
\end{aligned}
$$

The explicit expressions for $A^{(1)}\left(\varepsilon^{2} ; a\right),\left(\tilde{B}_{1}^{(1)} \pm \tilde{B}_{2}^{(1)}\right)\left(\bar{p}^{2} ; \varepsilon^{2}\right)$ and $\left(A^{(2)}+\tilde{B}^{(2)}\right)\left(\bar{p}^{2} ; \varepsilon^{2}\right)$ for the PTC and PV cases can therefore be read off directly from (B.4)-(B.5) [or equivalently from: (B.14) and (32)] and from (B.20)-(B.21), (33)-(34). With the exception of $A^{(1)}$, they are formally $\mathcal{O}\left(N_{\mathrm{c}}^{0}\right) ; A^{(1)}$ is $\mathcal{O}\left(1 / N_{\mathrm{C}}\right)$.

The general leading- $N_{\mathrm{c}}$ gap equation is obtained by minimizing the potential $\Xi^{(0)}$ with respect to $\varepsilon^{2}$

$$
\left.\frac{\partial \Xi^{(0)}\left(\varepsilon^{2} ; a\right)}{\partial \varepsilon^{2}}\right|_{\varepsilon^{2}=\varepsilon_{0}^{2}}=\frac{1}{a}-\left.\int_{0}^{\infty} \frac{d z}{z^{2}} \operatorname{reg}_{\mathrm{f}}(z) e^{-z \varepsilon^{2}}\right|_{\varepsilon^{2}=\varepsilon_{0}^{2}}=0
$$

where $\varepsilon_{0}=m_{t}^{(0)} / \Lambda_{\mathrm{f}}$, and $m_{t}^{(0)}$ is the top quark mass approximation as obtained from this leading- $N_{\mathrm{c}}$ gap equation. From (40) we also see explicitly that the dimensionless coupling constant $a$, as defined by (35), is really of order 1 .

At this point, we note that the NTL contribution in (38) is ill-defined for all $\varepsilon^{2}$ 's that are smaller than the leading- $N_{\mathrm{c}}$ gap equation solution $\varepsilon_{0}^{2}=\left(m_{t}^{(0)} / \Lambda_{\mathrm{f}}\right)^{2}$. The reason for this is that, as already mentioned in the previous Section, $\alpha_{1}$ and hence $A^{(1)}$ become negative for $\varepsilon^{2}<\varepsilon_{0}^{2}$, while $\tilde{B}_{1}^{(1)}-\tilde{B}_{2}^{(1)}$ and $A^{(2)}+\tilde{B}^{(2)}$, being nonnegative always, go to zero when $\bar{p}^{2} \rightarrow 0$, as seen from (B.17)-(B.18). Therefore, the arguments of the logarithms for the Goldstone contributions in (38) become negative in such a case. This problem is manifest already in formula (25) where the argument in the exponent 
in the integral over the sources $I_{j}$ becomes positive for $\varepsilon^{2}<\varepsilon_{0}^{2}$ (for $j=1,2,3$ ) and the integral becomes divergent. Furthermore, for the same reasons, the argument of the logarithm for the Higgs contribution in $(38)$ becomes negative for small $\varepsilon^{2}<\varepsilon_{*}^{2}$ (substantially smaller than $\varepsilon_{0}^{2}$ ) when $\bar{p}^{2} \rightarrow 0$.

As mentioned in the previous Section, however, the problematic part $A^{(1)}\left(\varepsilon^{2} ; a\right)$ of the arguments of the logarithms in the NTL part (38) of the effective potential is formally suppressed by $\left(1 / N_{\mathrm{c}}\right)$ in comparison to the other parts. Therefore, we may be tempted to simply ignore that term there, on the grounds that it gives formally the next-to-next-to-leading order (NNTL) corrections which have not been taken into account in its entirety here anyway. However, this argument is dangerous and misleading, because the physically relevant relations are obtained from the gap equation involving the derivative of the effective potential, and not the effective potential itself. It is straightforward to see from (37) and (40) that the derivative $\partial A^{(1)} / \partial \varepsilon^{2}=2 \partial^{2} \Xi^{(0)} / \partial\left(\varepsilon^{2}\right)^{2}$ at $\varepsilon^{2}=\varepsilon_{0}^{2}\left(1+\mathcal{O}\left(1 / N_{\mathrm{c}}\right)\right)$ is not suppressed by $1 / N_{\mathrm{c}}$, unlike $A^{(1)}$. In other words, $\partial A^{(1)} / \partial \varepsilon^{2}$ is $\mathcal{O}\left(N_{\mathrm{c}}^{0}\right)$ just like $\partial\left(\tilde{B}_{1}^{(1)} \pm \tilde{B}_{2}^{(1)}\right) / \partial \varepsilon^{2}$ and $\partial\left(A^{(2)}+\tilde{B}^{(2)}\right) / \partial \varepsilon^{2}$ are. Therefore, we are forced to keep this derivative in the NTL gap equation 1 . If we ignored $A^{(1)}$ in $\Xi^{(1)}$, we would lose its derivative in the gap equation, and would thus lose an important part of the NTL effects there. On these grounds, we get the NTL gap equation in the following form which is now free of any singularities and is thus numerically well defined:

$$
\begin{aligned}
& \frac{\partial \Xi_{\mathrm{eff}}\left(\varepsilon^{2} ; a\right)}{\partial \varepsilon^{2}}=\frac{1}{a}-\int_{0}^{\infty} \frac{d z}{z^{2}} \operatorname{reg}_{\mathrm{f}}(z) e^{-z \varepsilon^{2}} \\
& \quad+\frac{1}{4 N_{\mathrm{c}}} \int_{0}^{\Lambda_{\mathrm{b}}^{2} / \Lambda_{\mathrm{f}}^{2}} d \bar{p}^{2} \bar{p}^{2}\left\{\left[\frac{\partial A^{(1)}\left(\varepsilon^{2} ; a\right)}{\partial \varepsilon^{2}}+\frac{\partial\left(\tilde{B}_{1}^{(1)}+\tilde{B}_{2}^{(1)}\right)\left(\bar{p}^{2} ; \varepsilon^{2}\right)}{\partial \varepsilon^{2}}\right]\left[\left(\tilde{B}_{1}^{(1)}+\tilde{B}_{2}^{(1)}\right)\left(\bar{p}^{2} ; \varepsilon^{2}\right)\right]^{-1}\right. \\
& \quad+\left[\frac{\partial A^{(1)}\left(\varepsilon^{2} ; a\right)}{\partial \varepsilon^{2}}+\frac{\partial\left(\tilde{B}_{1}^{(1)}-\tilde{B}_{2}^{(1)}\right)\left(\bar{p}^{2} ; \varepsilon^{2}\right)}{\partial \varepsilon^{2}}\right]\left[\left(\tilde{B}_{1}^{(1)}-\tilde{B}_{2}^{(1)}\right)\left(\bar{p}^{2} ; \varepsilon^{2}\right)\right]^{-1} \\
& \left.\quad+2\left[\frac{\partial A^{(1)}\left(\varepsilon^{2} ; a\right)}{\partial \varepsilon^{2}}+\frac{\partial\left(A^{(2)}+\tilde{B}^{(2)}\right)\left(\bar{p}^{2} ; \varepsilon^{2}\right)}{\partial \varepsilon^{2}}\right]\left[\left(A^{(2)}+\tilde{B}^{(2)}\right)\left(\bar{p}^{2} ; \varepsilon^{2}\right)\right]^{-1}\right\}=0 .
\end{aligned}
$$

The fact that $\tilde{B}_{1}^{(1)}-\tilde{B}_{2}^{(1)}$ and $A^{(2)}+\tilde{B}^{(2)}$ are proportional to $\bar{p}^{2}$ when $\bar{p}^{2} \rightarrow 0$ is in (41) a manifestation of Goldstone theorem (masslessness of Goldstones). We will denote the solution to the NTL gap equation simply as: $\varepsilon^{2}=\varepsilon_{\text {gap }}^{2}$, or $\left(\varepsilon_{\text {gap }}^{(\mathrm{NTL})}\right)^{2}$. This NTL gap equation is "exact" in the sense that it includes all the NTL effects of the composite scalars (Higgs and Goldstones). This means that it includes also the contributions of the longitudinal components of the electroweak gauge bosons (in the

${ }^{4}$ On the other hand, we are allowed to ignore $A^{(1)}=\mathcal{O}\left(1 / N_{\mathrm{C}}\right)$ in the NTL gap equation; in fact, we must do this because otherwise the three integrands in the NTL part of (41) would have singularities [cf. discussion following Eqs. (31) and (40)] and Goldstone theorem would be violated. 
Landau gauge); it does not include the effects of the other - transverse - components of these gauge bosons. It does not yet include the effects of the gluons - this will be done in the next Section. The NTL gap equation (41) can be solved numerically, for any given value of $\Lambda_{\mathrm{b}}^{2} / \Lambda_{\mathrm{f}}^{2}$ and any allowed given value of the input parameter $a[a>1$ for the PTC, and $a>1 /(2 \ln 2)$ for the PV case].

There exists an alternative way of solving this equation - namely in the " $\left(1 / N_{\mathrm{c}}\right)$ perturbative" approach. We can make for the solution $\varepsilon_{\text {gap }}^{2}$ the familiar $1 / N_{\mathrm{c}}$ expansion ansatz

$$
\begin{gathered}
\left.\frac{\partial \Xi_{\mathrm{eff}}\left(\varepsilon^{2} ; a\right)}{\partial \varepsilon^{2}}\right|_{\varepsilon^{2}=\varepsilon_{\text {gap }}^{2}}=0, \\
\text { with: } \quad \varepsilon_{\text {gap }}^{2}=\frac{m_{t}^{2}(\Lambda)}{\Lambda_{\mathrm{f}}^{2}}=\varepsilon_{0}^{2}+\frac{1}{N_{\mathrm{c}}} \kappa_{1 \mathrm{~g}}+\mathcal{O}\left(\frac{1}{N_{\mathrm{c}}^{2}}\right),
\end{gathered}
$$

where $\varepsilon_{0}^{2}$ is the solution of the leading- $N_{\mathrm{c}}$ gap equation. Inserting expansion (43) into (42), using expansion (36) for $\Xi_{\text {eff }}$, and demanding that coefficients at each power of $1 / N_{\mathrm{c}}$ be zero, we obtain the following relations:

$$
\left.\frac{\partial \Xi^{(0)}}{\partial \varepsilon^{2}}\right|_{\varepsilon^{2}=\varepsilon_{0}^{2}}=0,\left.\quad \kappa_{1 \mathrm{~g}} \frac{\partial^{2} \Xi^{(0)}}{\partial\left(\varepsilon^{2}\right)^{2}}\right|_{\varepsilon^{2}=\varepsilon_{0}^{2}}+\left.\frac{\partial \Xi^{(1)}}{\partial \varepsilon^{2}}\right|_{\varepsilon^{2}=\varepsilon_{0}^{2}}=0 .
$$

The "( $\left.1 / N_{\mathrm{c}}\right)$ perturbative" NTL gap equation (44) determines the approximate change of the ratio $\varepsilon_{\text {gap }}^{2}=m_{t}^{2}(\Lambda) / \Lambda_{\mathrm{f}}^{2}$ due to NTL effects

$$
\delta\left(\varepsilon^{2}\right)_{\text {gap }}^{(\mathrm{NTL})}=\frac{\kappa_{1 \mathrm{~g}}}{N_{\mathrm{c}}}=-\left[\left.\frac{\partial \Xi^{(1)}}{\partial \varepsilon^{2}}\right|_{\varepsilon^{2}=\varepsilon_{0}^{2}}\right] /\left[\left.N_{\mathrm{c}} \frac{\partial^{2} \Xi^{(0)}}{\partial\left(\varepsilon^{2}\right)^{2}}\right|_{\varepsilon^{2}=\varepsilon_{0}^{2}}\right] .
$$

Incidentally, in Eq. (45) we do not have to worry about setting the problematic terms $A^{(1)}\left(\varepsilon^{2} ; a\right)$ to zero, since they are zero automatically for $\varepsilon^{2}=\varepsilon_{0}^{2}$. This latter approach was also applied in Ref. [20], where we employed the spherical covariant cutoff. We also discussed there briefly the problem of singularities that would occur otherwise. These problems of singularities in the NTL derivatives $\partial \Xi^{(1)} / \partial \varepsilon^{2}$ at $\varepsilon^{2}<\varepsilon_{0}^{2}$ have also been discussed by the authors of Ref. [28], in the context of an $S U(2)$ invariant NJL model which they regarded as a model for low energy QCD.

Expression (45) is a reasonably good approximation to the actual NTL change as determined by the "exact" NTL gap equation (41) only as long as the values of $\left|\delta\left(\varepsilon^{2}\right)_{\text {gap }}^{(\mathrm{NTL})} / \varepsilon_{0}^{2}\right|, \varepsilon_{0}^{2}$, and $\Lambda_{\mathrm{b}}^{2} / \Lambda_{\mathrm{f}}^{2}$ are all sufficiently small $(\lesssim 0.5)$. Even in such cases, expression $(45)$ can frequently overestimate $\left|\delta\left(\varepsilon^{2}\right)_{\text {gap }}^{(\mathrm{NTL})}\right|$ by more than ten percent. This expression is less precise in the PV cases than in the corresponding PTC and S cases. For all these reasons, we use the "exact" NTL gap equation (41) in our calculations.

In this context, we mention that it is possible also in the case of the covariant spherical cutoff (S) of fermionic momenta to write down the "exact" NTL gap equation free of any singularities, in 
close analogy with (41). The formal expression for $\partial \Xi^{(1)} / \partial \varepsilon^{2}$ in the $\mathrm{S}$ case was obtained in Ref. 20. That expression contains as integrands the S-regulated bubble-chain-corrected scalar propagators $a[1-$ $\left.a \mathcal{J}_{X}\left(\bar{p}^{2} ; \varepsilon^{2}\right)\right]^{-1} / 2(X=H, G n, G c h)$ f, which are represented in Fig. 3 as dashed lines with a blob. Their analogues in the proper time approach are the inverses of the arguments of logarithms in (38): $\left[\left(A^{(1)}+\tilde{B}_{1}^{(1)} \pm \tilde{B}_{2}^{(1)}\right)\left(\bar{p}^{2} ; \varepsilon^{2}\right)\right]^{-1}$ and $\left[\left(A^{(1)}+A^{(2)}+\tilde{B}^{(2)}\right)\left(\bar{p}^{2} ; \varepsilon^{2}\right)\right]^{-1}$. The S-regulated scalar propagators as integrands for $\partial \Xi^{(1)} / \partial \varepsilon^{2}$ have the same kind of singularities as their analogues in the proper time framework: for $X=G n$, Gch singularities occur for $\varepsilon^{2}<\varepsilon_{0}^{2}$; for $X=H$ singularities occur for $\varepsilon^{2}<\varepsilon_{*}^{2}\left(<\varepsilon_{0}^{2}\right)$. The trick now is to replace them in $\partial \Xi^{(1)} / \partial \varepsilon^{2}$ by $\left[1-\varepsilon^{2} \ln \left(\varepsilon^{-2}+1\right)-\mathcal{J}_{X}\left(\bar{p}^{2} ; \varepsilon^{2}\right)\right]^{-1} / 2$, and the difference between the inverse of the old and of the latter expression is equal to $2 \partial \Xi^{(0)} / \partial \varepsilon^{2}$, as was also the case in the proper time approach. This represents formally a next-to-next-to-leading (NNTL) modification, which is again completely legitimate at the level of the NTL gap equation. The resulting modified inverse propagators of the scalars can be shown to be positive everywhere, and therefore the NTL gap equation written below is free of any singularities:

$$
\begin{aligned}
& {\frac{\partial \Xi_{\mathrm{eff}}\left(\varepsilon^{2} ; a\right)}{\partial \varepsilon^{2}}}^{(\mathrm{S})}=\frac{1}{a}-\left[1-\varepsilon^{2} \ln \left(\varepsilon^{-2}+1\right)\right] \\
& \quad-\frac{1}{4 N_{\mathrm{c}}} \int_{0}^{\Lambda_{\mathrm{b}}^{2} / \Lambda_{\mathrm{f}}^{2}} d \bar{p}^{2} \bar{p}^{2}\left\{\sum_{X} A_{X} \frac{\partial \mathcal{J}_{X}\left(\bar{p}^{2} ; \varepsilon^{2}\right)}{\partial \varepsilon^{2}}\left[1-\varepsilon^{2} \ln \left(\varepsilon^{-2}+1\right)-\mathcal{J}_{X}\left(\bar{p}^{2} ; \varepsilon^{2}\right)\right]^{-1}\right\}=0 .
\end{aligned}
$$

The subscripts in the sum above are: $X=H, G n, G c h$; and the respective multiplicity factors are: $A_{X}=1,1,2$. The corresponding dimensionless two-point Green functions with the fermionic S-cutoff are

$$
\begin{aligned}
\mathcal{J}_{H}\left(\bar{p}^{2} ; \varepsilon^{2}\right) & =\frac{1}{\pi^{2}} \int_{\bar{k}^{2} \leq 1} d^{4} \bar{k} \frac{\left[\bar{k} \cdot(\bar{p}+\bar{k})-\varepsilon^{2}\right]}{\left(\bar{k}^{2}+\varepsilon^{2}\right)\left[(\bar{p}+\bar{k})^{2}+\varepsilon^{2}\right]}, \\
\mathcal{J}_{G n}\left(\bar{p}^{2} ; \varepsilon^{2}\right) & =\frac{1}{\pi^{2}} \int_{\bar{k}^{2} \leq 1} d^{4} \bar{k} \frac{\left[\bar{k} \cdot(\bar{p}+\bar{k})+\varepsilon^{2}\right]}{\left(\bar{k}^{2}+\varepsilon^{2}\right)\left[(\bar{p}+\bar{k})^{2}+\varepsilon^{2}\right]}, \\
\mathcal{J}_{G c h}\left(\bar{p}^{2} ; \varepsilon^{2}\right) & =\frac{1}{\pi^{2}} \int_{\bar{k}^{2} \leq 1} d^{4} \bar{k} \frac{\bar{k} \cdot(\bar{p}+\bar{k})}{\left(\bar{k}^{2}+\varepsilon^{2}\right)(\bar{p}+\bar{k})^{2}},
\end{aligned}
$$

and their explicit expressions were given in Ref. [20]. Also in the S case, we don't use the approximate NTL gap equation (45), but the "exact" S-cutoff NTL gap equation (46).

\footnotetext{
${ }^{5}$ Subscripts H, Gn and Gch correspond to NTL contributions from the Higgs, neutral Goldstone and charged Goldstone degrees of freedom, respectively.
} 


\section{Mass renormalization, QCD corrections}

We calculate and include at this point also the top quark mass renormalization effects $m_{t}(\Lambda) \mapsto m_{t}^{\text {ren. }}$. In this context, we stress that the hard mass of the NTL gap equation (41) is the fixed (nonrunning) mass in the dynamically broken effective theory (1) with a cutoff $\Lambda \sim \Lambda_{\mathrm{f}} \sim \Lambda_{\mathrm{b}}$, i.e., it is the bare mass $m_{t}(\Lambda)\left(\varepsilon_{\text {gap }}=m_{t}(\Lambda) / \Lambda_{\mathrm{f}}\right)$. In order to obtain the values of the cutoff parameters $\Lambda_{\mathrm{f}}$ and $\Lambda_{\mathrm{b}}$, we therefore have to perform, after having solved the NTL gap equation $\left(\mapsto m_{t}(\Lambda) / \Lambda_{\mathrm{f}}\right)$, also the mass renormalization $\left(m_{t}(\Lambda) / \Lambda_{\mathrm{f}} \mapsto m_{t}^{\text {ren. }} / \Lambda_{\mathrm{f}}\right)$, since the mass of the top quark is more or less known $\left(m_{t}^{\text {ren. }} \approx 180 \mathrm{GeV}\right)\left[29\right.$. It is straightforward to check that there are no leading- $N_{\mathrm{c}}$ contributions to these renormalization corrections, and that only the 1-PI diagrams shown in Fig. 3 account for the NTL renormalization effects (cf. also [19], 20] ). Therefore

$$
\delta\left(\varepsilon^{2}\right)_{\text {ren. }}=\frac{\left(m_{t}^{2}\right)_{\text {ren. }}}{\Lambda_{\mathrm{f}}^{2}}-\frac{m_{t}^{2}\left(\Lambda_{\mathrm{f}}\right)}{\Lambda_{\mathrm{f}}^{2}}\left(=\varepsilon_{\mathrm{ren} .}^{2}-\varepsilon_{\text {gap }}^{2}\right)=\frac{1}{N_{\mathrm{c}}} \kappa_{1 \mathrm{r}}+\mathcal{O}\left(\frac{1}{N_{\mathrm{c}}^{2}}\right)
$$

There are three separate contributions, coming from the Higgs, neutral Goldstone and the charged Goldstone "coated" (i.e., bubble-chain-corrected) propagators of Fig. 3

$$
\delta\left(\varepsilon^{2}\right)_{\mathrm{ren} .}^{(\mathrm{NTL})}=\frac{1}{N_{\mathrm{c}}} \kappa_{1 \mathrm{r}}=\frac{1}{N_{\mathrm{c}}}\left(\kappa_{1 \mathrm{r}}^{(H)}+\kappa_{1 \mathrm{r}}^{(G n)}+\kappa_{1 \mathrm{r}}^{(G c h)}\right) .
$$

In order to obtain the corresponding "coated" propagators of the scalars within the framework of the discussed proper time regularized results, we note that the two-point 1PI Green function of the scalar $\tilde{\sigma}_{j}$ in the Euclidean $\bar{x}$ space is

$$
\Gamma_{j}^{(2)}\left(\bar{x}_{2}-\bar{x}_{1}\right)=\frac{g}{2}\left[\frac{\delta}{\delta s_{j}\left(\bar{x}_{2}\right)} \frac{\delta}{\delta s_{j}\left(\bar{x}_{1}\right)} \triangle \Gamma\left(\left\{\sigma_{k} ; s_{k}\right\}\right)\right], \quad\left(g=G M_{0}^{2}\right)
$$

where $\triangle \Gamma\left(\left\{\sigma_{k} ; s_{k}\right\}\right)$ is the part of the scalar action that is quadratic in the scalar fluctuations $s_{k}(\bar{y})$. Incidentally, the scalar action is the expression in the curly brackets of the exponent in formula (23). These two-point Green functions are therefore proportional to the action kernels (29) (multiplied by $g / 2$ ). In the momentum space, the propagators are inversely proportional to the Fourier transforms of the above expressions

$$
\begin{aligned}
& \frac{(-i)}{\tilde{\Gamma}_{0}^{(2)}\left(\bar{p}^{2} ; \sigma_{0}^{2}\right)}=\frac{-2 i}{g}\left[\alpha^{(1)}\left(\sigma_{0}^{2}\right)+\left(\tilde{\beta}_{1}^{(1)}+\tilde{\beta}_{2}^{(1)}\right)\left(\bar{p}^{2} ; \sigma_{0}^{2}\right)\right]^{-1}, \\
& \frac{(-i)}{\tilde{\Gamma}_{1}^{(2)}\left(\bar{p}^{2} ; \sigma_{0}^{2}\right)}=\frac{-2 i}{g}\left[\alpha^{(1)}\left(\sigma_{0}^{2}\right)+\left(\tilde{\beta}_{1}^{(1)}-\tilde{\beta}_{2}^{(1)}\right)\left(\bar{p}^{2} ; \sigma_{0}^{2}\right)\right]^{-1}, \\
& \frac{(-i)}{\tilde{\Gamma}_{2}^{(2)}\left(\bar{p}^{2} ; \sigma_{0}^{2}\right)}=\frac{-2 i}{g}\left[\alpha^{(1)}\left(\sigma_{0}^{2}\right)+\alpha^{(2)}\left(\sigma_{0}^{2}\right)+\tilde{\beta}^{(2)}\left(\bar{p}^{2} ; \sigma_{0}^{2}\right)\right]^{-1}=\frac{(-i)}{\tilde{\Gamma}_{3}^{(2)}\left(\bar{p}^{2} ; \sigma_{0}^{2}\right)} .
\end{aligned}
$$


Using these "coated" scalar propagators in the diagrams of Fig. 3 in Euclidean space, and again setting the term $\alpha^{(1)}\left(\sigma_{0}^{2}\right)\left[\propto A^{(1)}\left(\varepsilon^{2}\right)\right]$ equal to zero since it represents formally NNTL effects and would otherwise give us singular integrals, we get the following expressions for the mass renormalization terms $\kappa_{1 \mathrm{r}}$ :

$$
\begin{aligned}
& \kappa_{1 \mathrm{r}}^{(H)}=-\frac{1}{2} \int_{0}^{\Lambda_{\mathrm{b}}^{2} / \Lambda_{\mathrm{f}}^{2}} \frac{d \bar{p}^{2}}{\left(\tilde{B}_{1}^{(1)}+\tilde{B}_{2}^{(1)}\right)\left(\bar{p}^{2} ; \varepsilon^{2}\right)}\left[\left(\sqrt{\bar{p}^{2}\left(\bar{p}^{2}+4 \varepsilon^{2}\right)}-\bar{p}^{2}\right)\left(2+\frac{\bar{p}^{2}}{2 \varepsilon^{2}}\right)-\bar{p}^{2}\right], \\
& \kappa_{1 \mathrm{r}}^{(G n)}=+\frac{1}{2} \int_{0}^{\Lambda_{\mathrm{b}}^{2} / \Lambda_{\mathrm{f}}^{2}} \frac{d \bar{p}^{2}}{\left(\tilde{B}_{1}^{(1)}-\tilde{B}_{2}^{(1)}\right)\left(\bar{p}^{2} ; \varepsilon^{2}\right)} \frac{1}{2 \varepsilon^{2}} \bar{p}^{2}\left[\bar{p}^{2}+2 \varepsilon^{2}-\sqrt{\bar{p}^{2}\left(\bar{p}^{2}+4 \varepsilon^{2}\right)}\right], \\
& \kappa_{1 \mathrm{r}}^{(G c h)}=\left\{\frac{1}{2} \int_{0}^{-\varepsilon^{2}} \frac{d \bar{p}^{2} \bar{p}^{2}\left[2+\bar{p}^{2} / \varepsilon^{2}\right]}{\left(A^{(2)}+\tilde{B}^{(2)}\right)\left(\bar{p}^{2} ; \varepsilon^{2}\right)}\right. \\
& -\frac{\varepsilon^{2}}{2} \int_{-\varepsilon^{2}}^{\Lambda_{\mathrm{b}}^{2} / \Lambda_{\mathrm{f}}^{2}} d \bar{p}^{2}\left[\frac{1}{\left(A^{(2)}+\tilde{B}^{(2)}\right)\left(\bar{p}^{2} ; \varepsilon^{2}\right)}-\frac{1}{\bar{p}^{2}} \lim _{\bar{q}^{\prime 2} \rightarrow 0}\left(\frac{{\overline{q^{\prime}}}^{2}}{\left(A^{(2)}+\tilde{B}^{(2)}\right)\left({\overline{q^{\prime}}}^{2} ; \varepsilon^{2}\right)}\right)\right] \\
& \left.-\frac{\varepsilon^{2}}{2} \lim _{{\overline{q^{\prime}}}^{\prime 2} \rightarrow 0}\left(\frac{{\overline{q^{\prime}}}^{2}}{\left(A^{(2)}+\tilde{B}^{(2)}\right)\left({\overline{q^{\prime}}}^{2} ; \varepsilon^{2}\right)}\right)\left[-\ln \varepsilon^{2}+\ln \left(\Lambda_{\mathrm{b}}^{2} / \Lambda_{\mathrm{f}}^{2}\right)\right]\right\} \text {. }
\end{aligned}
$$

Here, we denoted by $\varepsilon^{2}$ the "bare" mass value $\varepsilon_{\text {gap }}^{2}=m_{t}^{2}(\Lambda) / \Lambda_{\mathrm{f}}^{2}$, i.e., the solution of the NTL gap equation. The expressions above were obtained by assuming first a (normalized) Euclidean momentum $\bar{q}^{2}>0$ for the external top quark line in the diagrams of Fig. 3. Then the analytic continuation to the (approximate) on-shell values $\bar{q}^{2}=-q^{2}=-\varepsilon^{2}\left[=-m_{t}^{2}(\Lambda) / \Lambda_{\mathrm{f}}^{2}\right]$ had to be performed. In the cases of $\kappa_{1 \mathrm{r}}^{(H)}$ and $\kappa_{1 \mathrm{r}}^{(G n)}$, this continuation is equivalent to the simple substitution in the Euclidean integrands: $\bar{q}^{2} \mapsto-\varepsilon^{2}$. The contribution $\kappa_{1 \mathrm{r}}^{(G c h)}$ of the charged Goldstones leading to (54) is somewhat more complicated due to the fact that the pole at $\bar{p}^{2}=0$ of the massless charged Goldstone generates a logarithmic branch cut in $\kappa_{1 \mathrm{r}}^{(G c h)}\left(\bar{q}^{2}\right)$ at the threshold value $\bar{q}^{2}=0$. The analytic continuation follows then from the usual prescription: $\ln \bar{q}^{2} \mapsto \ln \left(-q^{2}-i \epsilon\right) \mapsto \ln q^{2}-i \pi$, for $q^{2}>0$. The real part of this term was written in (54) as a separate $\ln \varepsilon^{2}$-term. None of the integrals in (54) is singular.

The expressions $\kappa_{j \mathrm{r}}^{(X)}$ for the case of the covariant spherical (S) cutoff were written in Ref. [20] where we substituted for $\varepsilon^{2}$ the leading- $N_{\mathrm{c}}$ gap equation solution $\varepsilon_{0}^{2}$, instead of the "bare" mass solution $\varepsilon_{\text {gap }}^{2}$ of the NTL gap equation. This was formally an acceptable approximation, because the renormalization contributions $\delta\left(\varepsilon^{2}\right)_{\mathrm{ren} .}^{(\mathrm{NTL})}$ are $1 / N_{\mathrm{c}}$ effects, as are the NTL gap contributions $\delta\left(\varepsilon^{2}\right)_{\text {gap }}^{(\mathrm{NTL})}=\varepsilon_{\text {gap }}^{2}-\varepsilon_{0}^{2}$. However, we investigate the borderline cases where the NTL gap contributions are large: $\left|\delta\left(\varepsilon^{2}\right)_{\text {gap }}^{(\mathrm{NTL})}\right| \lesssim \varepsilon_{0}^{2}$, in general larger than $\left|\delta\left(\varepsilon^{2}\right)_{\text {ren. }}^{(\mathrm{NTL})}\right|$. Therefore, it would be more realistic also in the $\mathrm{S}$ case of 20 to insert in the integrands for $\kappa_{j \mathrm{r}}^{(X)}$, s the "bare" mass solution of 
the NTL gap equation (46): $\varepsilon^{2}=\varepsilon_{\text {gap }}^{2}\left(<\varepsilon_{0}^{2}\right)$. However, once we do that, the formulas for $\kappa_{j \mathrm{r}}^{(X)}$, $\mathrm{s}$ ( $X=G n, G c h)$ become singular. As mentioned at the end of the previous Section, the remedy for this formal problem is known: the replacement of the "coated" (bubble-chain-corrected) S-regularized scalar propagators $\left(1-a \mathcal{J}_{X}\right)^{-1}$ by $a^{-1}\left[1-\varepsilon^{2} \ln \left(\varepsilon^{-2}+1\right)-\mathcal{J}_{X}\right]^{-1}$, resulting formally in an NNTL modification (thus legitimate at the NTL level) and giving the completely nonsingular integrals for $\kappa_{j \mathrm{r}}^{(X)}$ 's in the S case:

$$
\begin{aligned}
& \kappa_{1 \mathrm{r}}^{(H)}=-\frac{1}{4} \int_{0}^{\Lambda_{\mathrm{b}}^{2} / \Lambda_{\mathrm{f}}^{2}} \frac{d \bar{p}^{2}}{\left[1-\varepsilon^{2} \ln \left(\varepsilon^{-2}+1\right)-\mathcal{J}_{H}\left(\bar{p}^{2} ; \varepsilon^{2}\right)\right]}\left[\left(\sqrt{\bar{p}^{2}\left(\bar{p}^{2}+4 \varepsilon^{2}\right)}-\bar{p}^{2}\right)\left(2+\frac{\bar{p}^{2}}{2 \varepsilon^{2}}\right)-\bar{p}^{2}\right], \\
& \kappa_{1 \mathrm{r}}^{(G n)}=+\frac{1}{4} \int_{0}^{\Lambda_{\mathrm{b}}^{2} / \Lambda_{\mathrm{f}}^{2}} \frac{d \bar{p}^{2}}{\left[1-\varepsilon^{2} \ln \left(\varepsilon^{-2}+1\right)-\mathcal{J}_{G n}\left(\bar{p}^{2} ; \varepsilon^{2}\right)\right]} \frac{1}{2 \varepsilon^{2}} \bar{p}^{2}\left[\bar{p}^{2}+2 \varepsilon^{2}-\sqrt{\bar{p}^{2}\left(\bar{p}^{2}+4 \varepsilon^{2}\right)}\right], \\
& \kappa_{1 \mathrm{r}}^{(G c h)}=+\frac{1}{4}\left\{\int_{0}^{-\varepsilon^{2}} \frac{d \bar{p}^{2} \bar{p}^{2}\left[2+\bar{p}^{2} / \varepsilon^{2}\right]}{\left[1-\varepsilon^{2} \ln \left(\varepsilon^{-2}+1\right)-\mathcal{J}_{G c h}\left(\bar{p}^{2} ; \varepsilon^{2}\right)\right]}\right. \\
& -\varepsilon^{2} \int_{-\varepsilon^{2}}^{\Lambda_{\mathrm{b}}^{2} / \Lambda_{\mathrm{f}}^{2}} d \bar{p}^{2}\left[\frac{1}{\left(1-\varepsilon^{2} \ln \left(\varepsilon^{-2}+1\right)-\mathcal{J}_{G c h}\left(\bar{p}^{2} ; \varepsilon^{2}\right)\right)}-\frac{2}{\bar{p}^{2} \ln \left(1+1 / \varepsilon^{2}\right)}\right] \\
& \left.-\frac{2 \varepsilon^{2}}{\ln \left(1+1 / \varepsilon^{2}\right)}\left[-\ln \varepsilon^{2}+\ln \left(\Lambda_{\mathrm{b}}^{2} / \Lambda_{\mathrm{f}}^{2}\right)\right]\right\} \text {. }
\end{aligned}
$$

We insert in these expressions the NTL "bare" mass value $\varepsilon^{2}=\varepsilon_{\text {gap }}^{2}$.

Finally, we include the leading part of QCD effects. This was already done in Refs. [19], [20] and we cite here only the results. The leading "gap" part of QCD is represented by the contributions coming from the diagrams of Fig. 2, where the internal dashed lines represent now the gluon propagators (in Landau gauge). Since QCD effects turn out to be only of minor numerical importance in our framework, we decided to regulate the corresponding QCD integrals by means of only one specific approach - by the proper time cutoff $1 / \Lambda_{\mathrm{f}}^{2}$ for the quarks and $1 / \Lambda_{\mathrm{b}}^{2}$ for the gluons. The corresponding contribution to $\Xi^{(1)}$ to be added in (38) was derived in [19]

$$
\Xi^{(1 ; \mathrm{gl})}\left(\varepsilon^{2} ; \Lambda_{\mathrm{b}}^{2} / \Lambda_{\mathrm{f}}^{2} ; a_{\mathrm{gl}}\right)=2 \int_{0}^{\Lambda_{\mathrm{b}}^{2} / \Lambda_{\mathrm{f}}^{2}} d \bar{p}^{2} \bar{p}^{2} \ln \left[1-a_{\mathrm{gl}} \mathcal{J}_{\mathrm{gl}}\left(\bar{p}^{2} ; \varepsilon^{2}\right)\right]
$$

Here, $a_{\mathrm{gl}}$ is the relevant QCD coupling parameter: $a_{\mathrm{gl}}=3 \alpha_{s}\left(m_{t}\right) / \pi \approx 0.105$. The (proper time regulated) two-point Green function $\mathcal{J}_{\text {gl }}$ appearing in (58) is

$$
\begin{aligned}
\mathcal{J}_{\mathrm{gl}}\left(\bar{p}^{2}, \varepsilon^{2}\right)= & \frac{1}{6} \ln \varepsilon^{2}+\frac{2}{9}-\left.\frac{1}{6}(2 w-1)\left[-2+\sqrt{4 w+1} \ln \left(\frac{\sqrt{4 w+1}+1}{\sqrt{4 w+1}-1}\right)\right]\right|_{w=\varepsilon^{2} / \bar{p}^{2}} \\
& -\frac{1}{6}\left(\frac{\bar{p}^{2}}{5}+\varepsilon^{2}\right)+\frac{1}{4}\left(\frac{\bar{p}^{4}}{140}+\frac{\bar{p}^{2} \varepsilon^{2}}{15}+\frac{\varepsilon^{4}}{6}\right)+\mathcal{O}\left(\bar{p}^{6}, \varepsilon^{6}\right) .
\end{aligned}
$$


It is worth mentioning that QCD expression (58), unlike (38), turns out to be numerically almost equal to its two-loop approximation (obtained by the replacement: $\ln \left[1-a_{\mathrm{gl}} \mathcal{J}_{\mathrm{gl}}\left(\bar{p}^{2}, \varepsilon^{2}\right)\right] \mapsto-a_{\mathrm{gl}} \mathcal{J}_{\mathrm{gl}}\left(\bar{p}^{2}, \varepsilon^{2}\right)$ ), the difference being only a fraction of a percent. This has to do with the small values of $a_{\mathrm{gl}}(E)$ at the relevant energies $E \gtrsim m_{t}$. That's why the QCD contributions to $\delta\left(\varepsilon^{2}\right)_{\text {gap }}$ turn out to be quite small (almost negligible) in comparison to the NTL contributions of the scalars in the present framework.

The leading QCD $m_{t}$-mass renormalization effect, which is numerically more important than the QCD contribution to $\delta\left(\varepsilon^{2}\right)_{\text {gap }}=\varepsilon_{\text {gap }}^{2}-\varepsilon_{0}^{2}$, comes from the version of the diagrams of Fig. 3, where the dashed line with a blob is now the gluonic propagator. With the proper time cutoff we obtain (cf. [19])

$$
\delta\left(\varepsilon^{2}\right)_{\text {ren. }}^{\mathrm{QCD}} \approx \frac{2}{3} a_{\mathrm{gl}} \varepsilon^{2}\left[\ln \left(\varepsilon^{-2}\right)+\ln \left(\Lambda_{\mathrm{b}}^{2} / \Lambda_{\mathrm{f}}^{2}\right)+0.256 \ldots+\frac{5 \Lambda_{\mathrm{f}}^{2}}{9 \Lambda_{\mathrm{b}}^{2}} \varepsilon^{2}+\mathcal{O}\left(\varepsilon^{4}\right)\right],
$$

where we insert again for $\varepsilon^{2}$ the "bare" mass value $\varepsilon_{\text {gap }}^{2}$. This expression is to be added to (49) in order to obtain the QCD modified $\delta\left(\varepsilon^{2}\right)_{\text {ren. }}=\varepsilon_{\text {ren. }}^{2}-\varepsilon_{\text {gap }}^{2}$.

In our original paper [19], we regarded only the scalar sector contributions to $V_{\text {eff }}$ and $\delta V_{\text {eff }} / \delta \varepsilon^{2}$ as being organized in an $1 / N_{\mathrm{c}}$ expansion, and assumed that QCD contributions can be organized in a perturbative series in powers of $\alpha_{s}\left(m_{t}\right)$. However, as argued in [2], [5] and [16], the dominant part of the QCD contributions is formally a leading- $N_{\mathrm{c}}$ contribution. The reason lies in the fact that $\alpha_{s}=\mathcal{O}\left(N_{\mathrm{c}}^{-1}\right)$. In $\Xi^{(1 ; \mathrm{gl})}$ in (58), the factor 2 is replaced in the case of a general $N_{\mathrm{c}}$ by $\left(N_{\mathrm{c}}^{2}-1\right) / 4=$ $\mathcal{O}\left(N_{\mathrm{c}}^{2}\right)$; furthermore, $\ln \left(1-a_{\mathrm{gl}} \mathcal{J}_{\mathrm{gl}}\right) \approx-a_{\mathrm{gl}} \mathcal{J}_{\mathrm{gl}} \propto a_{\mathrm{gl}} \propto \alpha_{s}=\mathcal{O}\left(N_{\mathrm{c}}^{-1}\right)$. Therefore, $\Xi^{(1 ; \mathrm{gl})}=\mathcal{O}\left(N_{\mathrm{c}}\right)$ when added to the NTL part $\Xi^{(1)}=\mathcal{O}\left(N_{\mathrm{c}}^{0}\right)$ of $(38)$. Hence, we see really that $\Xi^{(1 ; \mathrm{gl})}$ is formally not NTL, but leading- $N_{\mathrm{c}}$ contribution to $\Xi_{\text {eff }}$ in the expansion (36). Also the QCD renormalization contribution (60) is formally leading- $N_{\mathrm{c}}$. Namely, the factor $2 / 3 \mathrm{in}(60)$ is in the general case replaced by $\left(N_{\mathrm{c}}^{2}-1\right) /\left(4 N_{\mathrm{c}}\right)=\mathcal{O}\left(N_{\mathrm{c}}\right)$, and since $a_{\mathrm{gl}}=\mathcal{O}\left(N_{\mathrm{c}}^{-1}\right)$, we have $\delta\left(\varepsilon^{2}\right)_{\text {ren. }}^{\mathrm{QCD}}=\mathcal{O}\left(N_{\mathrm{c}}^{0}\right)$, i.e., formally leading- $N_{\mathrm{c}}$ effect. However, it will turn out that for the cases considered in the present paper (with cutoffs $\Lambda \sim 1 \mathrm{TeV}$ when the NTL contributions of the scalars become comparable to the leading- $N_{\mathrm{c}}$ quark loop contributions), QCD contributions are numerically almost an order of magnitude smaller than both the leading- $N_{\mathrm{c}}$ quark loop contributions and the NTL scalar contributions. That's why we included in our formulas QCD contributions in the NTL parts.

\section{$5 \quad$ Numerical evaluations}

The inputs for the integrations are the values of the parameter $a=N_{\mathrm{c}} G \Lambda_{\mathrm{f}}^{2} / 8 \pi^{2}$ of (35), which is essentially a dimensionless measure of the strength of the original four-fermion coupling $G$ in (1), 
as well as the values of the ratio $\Lambda_{\mathrm{b}} / \Lambda_{\mathrm{f}}(\sim 1)$ of the bosonic and fermionic cutoff parameters. The diagrams of Fig. 2 suggest $\bar{p}_{\text {max }}^{2} \leq \bar{k}_{\text {max }}^{2}$, implying the input values $\Lambda_{\mathrm{b}} / \Lambda_{\mathrm{f}} \lesssim 1$, at least in the more intuitive cases of the fermionic S or PTC cutoff. Therefore, we have made three input choices for these ratios in the PTC case: $\Lambda_{\mathrm{b}} / \Lambda_{\mathrm{f}}^{(\mathrm{PTC})}=0.5,1 / \sqrt{2}(\approx 0.707), 1$. In fact, it will turn out that $\Lambda_{\mathrm{b}} / \Lambda_{\mathrm{f}}^{(\mathrm{PTC})}>1$ does not lead to physically acceptable results.

It turns out that both the NTL gap equation (41) [with QCD contributions from (58) included] and the (NTL) mass renormalization effects (49), (52)-(54), (60) decrease the ratio $\varepsilon^{2}$ when compared to the leading- $N_{\mathrm{c}}$ value $\varepsilon_{0}^{2}$ of $(40)$. In other words, we have: $\delta\left(\varepsilon^{2}\right)_{\text {gap }}=\varepsilon_{\text {gap }}^{2}-\varepsilon_{0}^{2}<0$ and $\delta\left(\varepsilon^{2}\right)_{\text {ren. }}=$ $\varepsilon_{\text {ren. }}^{2}-\varepsilon_{\text {gap }}^{2}<0$. In general, we have $\left|\delta\left(\varepsilon^{2}\right)_{\text {gap }}\right|>\left|\delta\left(\varepsilon^{2}\right)_{\text {ren. }}\right|$. When $\varepsilon_{\text {gap }}^{2} \rightarrow 0, \delta\left(\varepsilon^{2}\right)_{\text {gap }}$ remains relatively stable while $\left|\delta\left(\varepsilon^{2}\right)_{\text {ren. }}\right|\left(<\varepsilon_{\text {gap }}^{2}\right) \rightarrow 0$. Hence, $\delta\left(\varepsilon^{2}\right)_{\text {gap }}$ is identified as the source of the observed " $1 / N_{\mathrm{C}^{-}}$ nonperturbative" behavior, unlike $\delta\left(\varepsilon^{2}\right)_{\text {ren. }}$, when $\varepsilon_{\text {gap }}^{2} \equiv m_{t}^{2}(\Lambda) / \Lambda_{\mathrm{f}}^{2} \rightarrow 0$. Therefore, if we require $1 / N_{\mathrm{c}}$ expansion in our framework to have at least some qualitatively predictive power, than the NTL gap change $\left|\delta\left(\varepsilon^{2}\right)_{\text {gap }}\right|$ should not be too large in comparison to $\varepsilon_{0}^{2}$, i.e., $m_{t}(\Lambda) / m_{t}^{(0)}=\sqrt{\varepsilon_{\text {gap }}^{2} / \varepsilon_{0}^{2}}$ should not decrease beyond some critical small value. Consequently, the leading- $N_{\mathrm{c}}$ ratio $\varepsilon_{0}^{2}$, or equivalently the input parameter $a$ [cf. (40)], should not decrease beyond certain corresponding critical values. In Table 1 , we chose in the PTC case, for given ratio $\Lambda_{\mathrm{b}} / \Lambda_{\mathrm{f}}$ (second column), various "critical" small values for $m_{t}^{2}(\Lambda) / m_{t}^{(0) 2}=\varepsilon_{\text {gap }}^{2} / \varepsilon_{0}^{2}: 1 / 4,1 / 3,1 / 2,2 / 3$ (third column). There is a certain arbitrariness in deciding which of these values represents most realistically the breakdown of the $1 / N_{\mathrm{c}}$ expansion approach in the PTC case. In the fourth column, the corresponding ratio of the renormalized mass vs. $m_{t}^{(0)}$ is given. The last two columns contain the corresponding values of the cutoff parameters $\Lambda_{\mathrm{b}}$ and $\Lambda_{\mathrm{f}}^{(\mathrm{PTC})}$, which are obtained from the calculated values of $\varepsilon_{\text {ren. }}^{2}=\left(m_{t}^{\text {ren. }} / \Lambda_{\mathrm{f}}\right)^{2}$ and the approximate experimental value $m_{t}^{\text {ren. }}=180 \mathrm{GeV}$ [29]. In the first column, the corresponding values of the coupling input parameter $a(\mathrm{PTC})$ are given. We note that the values in the last two columns are the upper bounds of the cutoff parameters, once we take the stand that the NTL gap equation effects should not be stronger than in the specific case, i.e., that the ratio $m_{t}(\Lambda) / m_{t}^{(0)}$ should not be smaller than the chosen critical value in the third column.

As we see from Table 1, in the discussed PTC case the cutoff parameters must be quite low, of order $1 \mathrm{TeV}$ or less, for $1 / N_{\mathrm{c}}$ expansion to have some predictive power. Furthermore, once we increase the cutoff parameter ratio $\Lambda_{\mathrm{b}} / \Lambda_{\mathrm{f}}$ to the value $1 / \sqrt{2} \approx 0.707$, or 1 , the value of the ratio $\varepsilon_{\text {gap }}^{2} / \varepsilon_{0}^{2}$ in the PTC case cannot even be larger than 0.545, 0.314, respectively, as displayed in Table 1. This shows that the larger ratios of $\Lambda_{\mathrm{b}} / \Lambda_{\mathrm{f}}$ lead to even larger NTL gap effects. Thus, for $\Lambda_{\mathrm{b}} / \Lambda_{\mathrm{f}}>1$, the ratio $\varepsilon_{\text {gap }}^{2} / \varepsilon_{0}^{2}$ in the PTC case is always smaller than 0.314 , indicating that $\Lambda_{\mathrm{b}}>\Lambda_{\mathrm{f}}$ is more or less 
unacceptable in the present framework, i.e., $1 / N_{\mathrm{c}}$ expansion loses predictability in this case.

In addition, in columns 5-6 we included the values of $m_{t}(\Lambda) / m_{t}^{(0)}$ and $m_{t}^{\text {ren. }} / m_{t}^{(0)}$ in the corresponding cases for the $\mathrm{PV}$, and in columns 7-8 for the $\mathrm{S}$ regularization. By the "corresponding values" we mean those values which correspond to the same values of the four-fermion coupling $G$ of Eq. (1) and the same values of the cutoff $\Lambda_{\mathrm{b}}$, since this parameter represents always the covariant spherical cutoff for the bosonic momenta and is not influenced by the regularization choice (PTC, $\mathrm{PV}, \mathrm{S})$ for the fermionic momenta. Technically, to find these corresponding values, i.e., to find the corresponding input parameters $y(X)=\Lambda_{\mathrm{b}} / \Lambda_{\mathrm{f}}(X)$ and $a(X)$ ( $X$ denotes PV or $\mathrm{S}$ ), we have to require in the numerical program that the two numbers $E_{1}=a(X) * y^{2}(X)=\Lambda_{\mathrm{b}}^{2} G N_{\mathrm{c}} /\left(8 \pi^{2}\right)$ and $E_{2}=a(X) * \varepsilon_{\text {ren. }}^{2}(X)=\left(m_{t}^{\text {ren. }}\right)^{2} G N_{\mathrm{c}} /\left(8 \pi^{2}\right)$ be the same as in the PTC case.

It should be mentioned that certain entries in Table 1 should be regarded with additional reservations - those for which the leading- $N_{\mathrm{c}}$ values $\varepsilon_{0}=m_{t}^{(0)} / \Lambda_{\mathrm{f}}$ are larger than 1 . These are the entries of the last line for the PTC case, the last three lines for the PV case, and for the S case the lines corresponding to $a(\mathrm{PTC})=5.091$ and 7.935. On the other hand, all the entries in Table 1 have the values of $\varepsilon_{\text {gap }}^{(\mathrm{NTL})}=m_{t}(\Lambda) / \Lambda_{\mathrm{f}}$ and $\varepsilon_{\text {ren. }}^{(\mathrm{NTL})}=m_{t}^{\text {ren. }} / \Lambda_{\mathrm{f}}$ smaller than 1 .

A few additional technical remarks: In the PV case, the series for $\left(\tilde{\beta}_{1}^{(1)} \pm \tilde{\beta}_{2}^{(1)}\right)$ in inverse powers of $\Lambda_{\mathrm{f}}$ [Eq. (33)] is, unfortunately, quite slowly convergent for low values of $\Lambda_{\mathrm{f}}(\leq 0.5 \mathrm{TeV})$, unlike the series for $\left(\alpha^{(2)}+\tilde{\beta}^{(2)}\right)$ [Eq. (34)]. Therefore, we used in our calculations in the PV case for $\left(\tilde{\beta}_{1}^{(1)} \pm \tilde{\beta}_{2}^{(1)}\right)$ the closed analytic expression (B.23). For $\left(\alpha^{(2)}+\tilde{\beta}^{(2)}\right)$ in the PV case we used the closed analytic expresssion (B.24), except in Eq. (54). In this equation for $\kappa_{1 \mathrm{r}}^{(G c h)}$, describing the mass renormalization effects due to the charged Goldstone degrees of freedom, a part of the integration is performed over a region with negative $\bar{p}^{2}$, for which formula (B.24) is not suitable. Therefore, we applied there in the PV case the series (34), including the terms in the sum up to $n=8$.

In all regularization cases, we are led to the same qualitative conclusion: the cutoff $\Lambda_{\mathrm{b}}$ does not surpass $\mathcal{O}(1 \mathrm{TeV})$ as long as we demand that the NTL gap effects not drastically "wash out" the leading- $N_{\mathrm{c}}$ effects. Comparing the ratios $\left(m_{t}(\Lambda) / m_{t}^{(0)}\right)=\sqrt{\varepsilon_{\text {gap }}^{2} / \varepsilon_{0}^{2}}$ for the corresponding cases of the PTC, PV and S regularization, as well as the ratios $\left(m_{t}^{\text {ren. }} / m_{t}^{(0)}\right)=\sqrt{\varepsilon_{\text {ren. }}^{2} / \varepsilon_{0}^{2}}$, we conclude the following:

- The $\mathrm{S}$ cases give somewhat smaller gap NTL changes $\left|\delta\left(\varepsilon^{2}\right)_{\text {gap }} / \varepsilon_{0}^{2}\right|$ of the solution of the gap equation than the corresponding PTC cases; this implies that the NTL-tolerable values of the cutoff parameter $\Lambda_{\mathrm{b}}$ are somewhat higher in the S cases. On the other hand, the full NTL changes $\left|\left[\delta\left(\varepsilon^{2}\right)_{\text {gap }}+\delta\left(\varepsilon^{2}\right)_{\text {ren. }}\right] / \varepsilon_{0}^{2}\right|$ are almost the same in both cases. 
- The PV cases give somewhat larger NTL changes $\left|\delta\left(\varepsilon^{2}\right)_{\text {gap }} / \varepsilon_{0}^{2}\right|$ than the corresponding PTC cases; this implies that the NTL-tolerable values of the cutoff parameter $\Lambda_{\mathrm{b}}$ are somewhat lower in the PV cases. Also the full NTL changes $1-\varepsilon_{\text {ren. }}^{2} / \varepsilon_{0}^{2}=\left|\left[\delta\left(\varepsilon^{2}\right)_{\text {gap }}+\delta\left(\varepsilon^{2}\right)_{\text {ren. }}\right] / \varepsilon_{0}^{2}\right|$ are somewhat lower in the PV cases.

When inspecting more closely the separate contributions of the various degrees of freedom to the NTL gap shift $\delta\left(\varepsilon^{2}\right)_{\text {gap }}=\varepsilon_{\text {gap }}^{2}-\varepsilon_{0}^{2}$ of (41) and to the mass renormalization NTL shift $\delta\left(\varepsilon^{2}\right)_{\text {ren. }}=$ $\varepsilon_{\text {ren. }}^{2}-\varepsilon_{\text {gap }}^{2}$ of (49), (52)-(54), (60), for the cases displayed in Table 1, we see the following f5 the Higgs and each one of the three Goldstone degrees of freedom contribute comparable negative values to $\delta\left(\varepsilon^{2}\right)_{\text {gap }}$, and gluons a small positive value which is by an order of magnitude smaller than the absolute values of the separate scalar contributions; the Higgs and the charged Goldstone degrees of freedom contribute each a negative value and the neutral Goldstone and gluons weaker positive values to $\delta\left(\varepsilon^{2}\right)_{\text {ren. }}$, resulting thus in a negative $\delta\left(\varepsilon^{2}\right)_{\text {ren. }}$ Therefore, both $\delta\left(\varepsilon^{2}\right)_{\text {gap }}$ and $\delta\left(\varepsilon^{2}\right)_{\text {ren. }}$ are negative, and $\left|\delta\left(\varepsilon^{2}\right)_{\text {gap }}\right|$ is usually larger than $\left|\delta\left(\varepsilon^{2}\right)_{\text {ren. }}\right|$ by more than a factor of 2 .

In Ref. [20] we calculated the NTL effects for the S case of regularization of fermionic momenta. Comparing them with the results of Table 1 for the $\mathrm{S}$ case, we find that the cutoffs $\Lambda_{\mathrm{b}}$ in Table 1 are somewhat larger in the corresponding cases. These differences arise because, unlike in [20], here we inserted in the nonsingular ("regularized") expressions of the renormalization contributions (55)-(57) and (60) the NTL corrected "bare" mass parameter $\varepsilon_{\text {gap }}^{2}$, as mentioned earlier, and not $\varepsilon_{0}^{2}\left(\varepsilon_{0}^{2}>\varepsilon_{\text {gap }}^{2}\right)$, resulting thus in numerically smaller numbers for $\delta\left(\varepsilon^{2}\right)_{\text {ren. }}=\varepsilon_{\text {ren. }}^{2}-\varepsilon_{\text {gap }}^{2}$. In 20], on the other hand, we used singular integral expressions for $\kappa_{1 \mathrm{r}}^{(X)}(X=H, G n, G c h)$, and we had no other choice there but to insert for $\varepsilon^{2}$ the value $\varepsilon_{0}^{2}$ to avoid singularities in the integrals over bosonic momenta. If we inserted $\varepsilon^{2}=\varepsilon_{0}^{2}$ in the nonsingular ("regularized") expressions (55)-(57), these would give us results for $\kappa_{1 \mathrm{r}}$ identical with those in [20], as it should be. In addition, in [20] we used the approximate NTL gap equation (45) to obtain $\delta\left(\varepsilon^{2}\right)_{\text {gap }}$, and not the "exact" NTL gap equation (46) for the S cutoff. This resulted in 20] in somewhat larger values for $\left|\delta\left(\varepsilon^{2}\right)_{\text {gap }}\right|$, and contributed thus additionally to smaller values of $\Lambda_{\mathrm{b}}$ (and $\Lambda_{\mathrm{f}}$ ). Incidentally, in [20] we regarded $\left(\delta\left(\varepsilon^{2}\right)_{\text {gap }}+\delta\left(\varepsilon^{2}\right)_{\text {ren. }}\right) / \varepsilon_{0}^{2}\left[\mapsto\left(m_{t}^{\text {ren. }} / m_{t}^{(0)}\right)^{2}\right]$ as a measure of the NTL changes. On the other hand, in the present paper we chose to regard only the NTL gap effects as the genuine NTL effects, i.e., we chose as a measure of the NTL changes the ratio $\delta\left(\varepsilon^{2}\right)_{\text {gap }} / \varepsilon_{0}^{2}\left[\mapsto\left(m_{t}(\Lambda) / m_{t}^{(0)}\right)^{2}\right]$.

\footnotetext{
${ }^{6}$ To estimate roughly the separate contributions to $\delta\left(\varepsilon^{2}\right)$ gap, we use the approximate NTL gap equation (45), where these contributions are purely additive.
} 


\section{Conclusions and comparison to other works}

In this work, we calculated the next-to-leading (NTL) terms in $1 / N_{\mathrm{c}}$ expansion of the effective potential of $\bar{t} t$ condensate and of the corresponding gap equation, in the effective non-gauged $S U(2)_{L} \times U(1)_{Y}$ Nambu-Jona-Lasinio type model (called also the top-mode standard model - TSM) of dynamical symmetry breaking (DSB). Furthermore, we calculated also the (NTL) mass renormalization effects after the DSB. We included all the degrees of freedom that are relevant at the NTL level of this framework: the Higgs and the three Goldstone condensates, and the quarks of the third generation (top and bottom). In addition, we included also the dominant part of QCD contributions. The latter turned out to be numerically less important in the present framework. We considered the effective potential as a function of a hard mass term $\sigma_{0}$ of the top quark, i.e., of the expectation value $\sigma_{0}$ of the composite scalar isodoublet field $\hat{\sigma}=\sqrt{g} \hat{\Phi}$. We concentrated in particular on the question of regularizing the integrals over the fermionic Euclidean momenta in a way that is mutually consistent at the leading- $N_{\mathrm{c}}$ and at the NTL level, and is free of the momentum branching ambiguities. These ends are achieved by employing the proper time regularization techniques, and we specifically employed the proper time cutoff (PTC) and the Pauli-Villars (PV) two-subtractions regulator for the fermionic momenta within the proper time framework. Furthermore, we discussed in detail how to ensure the validity of Goldstone theorem at the NTL level - in the proper time regularization framework and in the case of the simple covariant spherical $(\mathrm{S})$ cutoff for the fermionic momenta. For integrals over the bosonic momenta, no branching ambiguity problem appeared, but the proper time approach doesn't regularize them. We always employed covariant spherical cutoff for them. The dependence of our results on the various regularization schemes (PTC, PV, S) for the fermionic momenta was investigated. The basic conclusions of the previous paper [20], in which a (simplified) S regularization approach was applied, remain unchanged: as long as the cutoff energy $\Lambda\left(\sim \Lambda_{\mathrm{f}} \sim \Lambda_{\mathrm{b}}\right)$, at which the $\bar{t} t$ condensation is assumed to take place, is larger than $\mathcal{O}(1 \mathrm{TeV})$, then the negative NTL gap corrections $\delta\left(\varepsilon^{2}\right)_{\text {gap }}$ to the DSB (the gap equation solution) have absolute values quite close to the values of the positive leading- $N_{\mathrm{c}}$ quark loop contributions $\varepsilon_{0}^{2}$, thus essentially "washing out" the latter ones and making the model difficult or impossible to interpret. On the other hand, for $\Lambda<\mathcal{O}(1 \mathrm{TeV})$, explicit calculations here show that the PTC, PV and S regularizations, when physically acceptable, give similar numerical results, the PV regularization having somewhat stronger and the S regularization somewhat weaker NTL gap corrections than the PTC in the corresponding cases.

As argued in the previous Section, we identify $\left|\delta\left(\varepsilon^{2}\right)_{\text {gap }}\right|$ as the source of the $1 / N_{\mathrm{c}}$ expansion breakdown, in contrast to the smaller $\left|\delta\left(\varepsilon^{2}\right)_{\text {ren. }}\right|$. The conclusion that the NTL gap contributions can 
easily become strong [for $\Lambda>\mathcal{O}(1 \mathrm{TeV})$ too strong for the applicability of $1 / N_{\mathrm{c}}$ expansion] is not quite implausible. The NTL contributions come primarily from the coupling of the composite scalar sector itself to its constituent top quarks (some kind of "feedback effect"). This coupling must be relatively strong because otherwise the condensation cannot occur in the first place.

The conclusions of this paper seem to contrast with those of Bardeen, Hill and Lindner (BHL) 《4 and Miransky, Tanabashi and Yamawaki (MTY) [2]; however, they don't necessarily exclude them. In the following, we briefly describe the approaches and results of BHL and MTY, compare them with our approach and results, and point out analogies, differences, and those points that remain unclear and deserve further investigation.

The authors of [4] employed the one-loop renormalization group equation (RGE) of the minimal SM for the top quark Yukawa coupling $g_{t}$ and demanded that it diverge or become large at the energy of condensation $\Lambda$. This is motivated by the compositeness condition which says that the renormalization constants of the composite scalar fields should vanish in a theory with the cutoff as high as $E=\Lambda$, i.e., that the composite particles disappear (disintegrate) at that energy. This RGE approach implicitly assumes that $\Lambda$ is large, i.e., that $\ln \left(\Lambda / E_{\text {ew }}\right) \gg 1$, and that the details of the condensation mechanism get decoupled from the minimal SM behavior at energies which are, on logarithmic scale, quite close to (but below) the energy $\Lambda$. Their approach results in very large $\Lambda$ 's; the larger the $\Lambda$, the smaller the $m_{t}^{\text {ren. }}$. For $\Lambda \sim \Lambda_{\text {Planck }}\left(\sim 10^{19} \mathrm{GeV}\right)$, they obtain $m_{t}^{\text {ren. }} \approx 220 \mathrm{GeV}$, still substantially higher than the measured $m_{t}^{\text {phys. }} \approx 170-180 \mathrm{GeV}\left[29\right.$. This approach concentrates on the $\delta\left(m_{t}\right)_{\text {ren. }}$ effects - the one-loop RGE for $g_{t}$ contains the leading- $N_{\mathrm{c}}$ (including the QCD) and at least one part of the NTL scalar contributions to $\delta\left(g_{t}\right)_{\text {ren. }}\left[\mapsto \delta\left(\varepsilon^{2}\right)_{\text {ren. }}\right]$ ]. The authors of [⿴囗⿰丿㇄口] argue that, due to the quasi infrared (IR) fixed-point behavior of the RGE, their prediction of $m_{t}^{\text {ren. }}=g_{t}^{\text {ren. }} v / \sqrt{2}$ is quite stable against any details of the actual condensation mechanism. More specifically, their $m_{t}^{\text {ren. }}$ is quite stable when $g_{t}^{2}(\Lambda) /(4 \pi)$ is changed between the values 1 and $\infty$, and/or $\ln \left(\Lambda / E_{\text {ew }}\right)$ is changed by values of $\mathcal{O}(1)$. This is reasonably true as long as $\Lambda>10^{10} \mathrm{GeV}$, limiting thus the applicability of their method to the scenarios with thus large cutoffs. Including the two-loop effects in the then coupled RGEs for $g_{t}$ and for the composite scalar self-coupling $\lambda$ doesn't change much the results of this method [14] - the predicted $m_{t}^{\text {ren. }}$ is then increased by a few $\mathrm{GeV}$.

The approach by MTY [2], and subsequently by King and Mannan [5], is closer to the approach

\footnotetext{
${ }^{7}$ If only the leading- $N_{c}$ quark loop effects are included in the RGE of the minimal SM, the running of the logarithms of the Yukawa coupling and the vacuum expectation value are exactly opposite to each other, so that $m_{t}$ is non-running. This is in agreement with the well known fact that the quark loop leading- $N_{\mathrm{C}}$ solution to the gap equation in TSM does not get modified by renormalization at that level of approximation.
} 
of the present paper. Unlike BHL, they investigate the actual condensation mechanism of TSM, by considering the Dyson-Schwinger (DS) integral equation for the top quark mass function $\Sigma_{t}\left(\bar{q}^{2}\right)$ and the Pagels-Stokar relation (PS) [30]. The DS equation was applied at the leading- $N_{\mathrm{c}}$ order (including QCD), and is basically the variational version of the usual gap equation; the gap equation at the leading- $N_{\mathrm{c}}$ order can be recovered from this DS equation by replacing $\Sigma_{t}\left(\bar{q}^{2}\right)$ by $m_{t}(\Lambda)$ and the oneloop running $\alpha_{s}\left(\bar{p}^{2}\right)$ by a constant $\alpha_{s}\left(E_{0}\right)\left(E_{0} \geq m_{t}\right)$. The mass function $\Sigma_{t}\left(\bar{q}^{2}\right)$ appears in the top quark propagator and is essentially the running mass of the top quark, in the non-perturbative sense.

In our approach, on the other hand, we took into account the effects of the running of mass $m_{t}\left(\bar{q}^{2}\right)$ between $\bar{q}^{2}=\Lambda_{\mathrm{f}}^{2}$ and the electroweak scale $E_{\mathrm{ew}}^{2}$ by calculating in an " $1 / N_{\mathrm{c}-\text { perturbative" }}$ way $\delta\left(\varepsilon^{2}\right)_{\text {ren. }}$ of QCD and the NTL scalar effects, but only after solving the NTL gap equation. However, unlike MTY, we didn't include in our investigation the PS relation or an NTL-improved analogous relation. PS relation contains leading- $N_{\mathrm{c}}$ effects of the Bethe-Salpeter (BS) bound state equation for the composite Goldstones of and connects the low energy vacuum expectation value $(\mathrm{VEV}) v \approx 246 \mathrm{GeV}$ (or equivalently: $M_{W}$ ) with $\Sigma_{t}\left(\bar{q}^{2}\right)$ and $\Lambda$. On the other hand, the DS (or: gap) equation connects the four-quark strength parameter $a=G N_{\mathrm{c}} \Lambda_{\mathrm{f}}^{2} /\left(8 \pi^{2}\right) \sim 1$ with $\Sigma_{t}^{2}\left(\bar{q}^{2}\right) / \Lambda_{\mathrm{f}}^{2}$ (or: $m_{t}^{2}(\Lambda) / \Lambda_{\mathrm{f}}^{2}=\varepsilon_{\text {gap }}^{2}$ ). Therefore, the combination of DS and BS predicts, for a given value of the

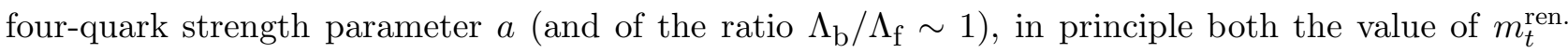
and of the onset scale $\Lambda\left(\sim \Lambda_{\mathrm{f}} \sim \Lambda_{\mathrm{b}}\right)$ of the underlying physics. Looking the other way around, since $m_{t}^{\text {phys. }}$ is experimentally known $\left(m_{t}^{\text {phys. }} \approx 170-180 \mathrm{GeV}\right)$, such calculations predict $\Lambda$ and $a(G)$.

MTY approach, as performed by [2] and [5] at the leading- $N_{\mathrm{c}}$ level, was shown to be numerically [15] and analytically [16] equivalent to the leading- $N_{\mathrm{c}}$ version of BHL approach. Furthermore, the results of BHL and the (leading- $N_{\mathrm{c}}$ ) results of MTY are relatively close to each other: $\left(m_{t}^{\text {ren. }}\right)_{\text {MTY }} \gtrsim 240 \mathrm{GeV}$ for $\Lambda \leq \Lambda_{\text {Planck. }}$. This would suggest that MTY approach could be continued to include NTL corrections for very large $\Lambda$ 's, if we trust BHL approach for such $\Lambda$ 's. On the other hand, the results of the present paper suggest that $1 / N_{\mathrm{c}}$ expansion fails for $\Lambda>\mathcal{O}(1 \mathrm{TeV})$. Therefore, the first question to be asked is: Would the modification of our approach, by changing the NTL gap equation (41) [with QCD effects from (58) included] to its variational version of MTY-type, change the basic conclusions of the paper? We have indications that it would not. Namely, the "running" of the mass function $\Sigma_{t}\left(\bar{q}^{2}\right)$ can be approximately described by varying $\varepsilon^{2}$ between $\varepsilon_{\text {gap }}^{2}\left(=m_{t}^{2}(\Lambda) / \Lambda_{\mathrm{f}}^{2}\right)$ and $\varepsilon_{\text {ren. }}^{2}\left(\approx m_{t}^{2}\left(E_{\text {ew }}\right) / \Lambda_{\mathrm{f}}^{2}\right)$. For the entries in Table 1 , however, these values differ roughly only by 5 , 10 and 20 percent, for $\Lambda_{\mathrm{f}} / \Lambda_{\mathrm{b}}=1 / 2,1 / \sqrt{2}, 1$, respectively. Also QCD contributions are quite small in

\footnotetext{
${ }^{8}$ The problem of incorporating systematically NTL effects in the BS equation has to our knowledge not been inves-
} tigated yet. 
all these cases.

However, our calculations do not exclude the possibility of much higher values of the cutoff $\Lambda$, i.e., values of several orders of magnitude larger than $1 \mathrm{TeV}$. The reason may lie in our separation of the calculation of the mass gap and of the mass renormalization effects. It is possible that this approach may not work well for very large $\Lambda$ 's $[\ln (\Lambda / 1 \mathrm{TeV}) \gg 1]$ where the interplay between the two effects may become important. In such a case, the variational (DS) version of the gap equation at the NTL level, which takes into account both the mass gap and the mass running effects simultaneously and thus also their interplay, would be the appropriate tool to apply. Then we could possibly see whether the scalar NTL corrections to $\varepsilon^{2}\left(\bar{q}^{2}\right)=\Sigma_{t}^{2}\left(\bar{q}^{2}\right) / \Lambda_{\mathrm{f}}^{2}$ for $\Lambda_{\mathrm{f}} \gg \mathcal{O}(1 \mathrm{TeV})$ can become tolerably small for $1 / N_{\mathrm{c}}$ expansion to make sense. One possible signal that our paper cannot exclude the entire region $\Lambda>\mathcal{O}(1 \mathrm{TeV})$ from the $1 / N_{\mathrm{c}}$ expansion approach lies in the fact that the leading QCD contributions to $\varepsilon_{\text {ren. }}^{2}$, while for $\Lambda \leq \mathcal{O}(1 \mathrm{TeV}$ ) being substantially smaller than the quark loop (bubblechain) contributions, start for much higher $\Lambda$ 's to compete with the bubble-chain contributions and substantially change the results in the DS + PS approach [16]. Therefore, we believe that performing the calculations at the NTL level with the variational (DS) approach would be important at this stage, in order to investigate the region of very high momenta. This could be done by employing the formalism of Cornwall, Jackiw and Tomboulis (CJT) [31] in which it is possible to calculate the effective potential as a functional of the top quark propagator, and hence of the mass function $\Sigma_{t}\left(\bar{q}^{2}\right)$. In addition, the inclusion of the BS equation at the NTL level would represent an additional important step in investigating the whole realm of the NTL effects of the strong composite scalar sector on the condensation mechanism.

One may also ask how the results would change when including the pure (i.e., transverse) components of the electroweak gauge bosons in the calculations. The RGE approach of BHL indicates that these contributions, at least as to the mass renormalization, are of minor importance, due to the relatively small $S U(2)_{L} \times U(1)_{Y}$ gauge couplings. Furthermore, the condensation occurs primarily due to the strong enough four-quark attractive coupling G of Eq. (1), so that the NTL contributions of the strong composite scalar sector to itself, i.e., the effects of the composite scalar couplings to its own constituent top quarks ("feedback effects"), are expected to be stronger and more important than those of the weak $S U(2)_{L} \times U(1)_{Y}$ couplings.

As a point independent of the discussion above, we stress that the simple TSM framework (11), in which the 6-dimensional four-quark contact term triggers the top quark condensation, might not be sufficient for a fully realistic picture when the energy $\Lambda$ at which the condensation takes place is as low 
as $\mathcal{O}(1 \mathrm{TeV})$. This is because higher dimensional femionic contact terms 32 33, which come from the details of the underlying physics at $E>\Lambda$, might contribute to the physical quantities relative corrections as high as $1 / \ln \left(\Lambda^{2} / m_{t}^{2}\right) \approx 0.3$. However, these corrections can be, for specific models of the underlying physics, substantially smaller [34].

Other authors have investigated NTL effects in NJL-type frameworks without gauge bosons [28], 35]- 38]. The authors of [28] calculated the NTL effects with an effective action formalism, and those of [35] with diagrammatic methods. Both groups regarded their discussed $S U(2)$ symmetric NJL type model as a framework of the low energy QCD and took particular care that Goldstone theorem remains valid.

The authors of [36] calculated NTL contributions to critical exponents of the fields at the fixed point, i.e., at the location of the nontrivial zero of $\beta$ function, for various dimensions $d \leq 4$. The implications of [36] for physical predictions of four-dimensional NJL models with finite cutoff are not clear from these works and would deserve investigation. On the other hand, Akama 37] investigated the NTL contributions by considering the compositeness condition which says that the renormalization constants of composite scalar fields and their self-interaction parameters should be zero. Also, Lurié and Tupper [38] had earlier investigated the compositeness condition, taking into account at least some of the NTL effects. Both Akama and Lurié and Tupper conclude that the compositeness condition implies that the NTL contributions to physical quantities for $N_{\mathrm{C}}=3$ are larger than the leading- $N_{\mathrm{c}}$ contributions, indicating that $1 / N_{\mathrm{c}}$ expansion diverges. We stress that these three authors treated TSM as a renormalizable Yukawa type model without gauge bosons plus the compositeness condition, an approach similar (not identical) to the approach of BHL [⿶]. Hence, implicitly they assumed that the cutoffs $\Lambda$ are large, i.e., that $\ln \Lambda$ terms entirely dominate over the $\Lambda$-independent terms. Therefore, their results apparently don't contradict the results of the present paper and [20] - i.e., that TSM without electroweak gauge bosons can make sense at the NTL level only if the cutoffs are quite low: $\Lambda \sim \Lambda_{\mathrm{f}} \sim \Lambda_{\mathrm{b}}=\mathcal{O}(1 \mathrm{TeV})$.

\section{Acknowledgment}

This work was supported in part by the Deutsche Forschungsgemeinschaft and in part by European Union Science Project No. SC1-CT91-0729. 


\section{Appendix A Diagonalization of $\hat{\mathcal{D}}^{\dagger} \hat{\mathcal{D}}\left(\left\{\sigma_{j}\right\}\right)$}

The non-negative definite hermitean operator $\hat{\mathcal{D}}^{\dagger} \hat{\mathcal{D}}\left(\left\{\tilde{\sigma}_{j}\right\}\right)$, in the Euclidean metric, is obtained directly from (4)-(5):

$$
\hat{\mathcal{D}}^{\dagger} \hat{\mathcal{D}}\left(\left\{\sigma_{j}\right\}\right)=\hat{\bar{P}} \cdot \hat{\bar{P}}++\hat{\mathcal{M}}^{\dagger} \hat{\mathcal{M}}+i \bar{\gamma}_{\mu} \frac{\partial}{\partial \bar{x}_{\mu}} \hat{\mathcal{M}}
$$

where we denoted the Euclidean quantities: $\bar{x}_{0}=i x^{0}, \bar{x}_{j}=x^{j}, \bar{\gamma}_{0}=i \gamma^{0}, \bar{\gamma}_{j}=\gamma^{j}$, and $\hat{\bar{P}}_{\mu}=i \partial / \partial \bar{x}_{\mu}$. The $8 \times 8$ matrix $\hat{\mathcal{M}}\left(\left\{\tilde{\sigma}_{j}\right\}\right)$ is the one written in Eq. (四). Since $\tilde{\sigma}_{j}(\bar{x})=\sigma_{j}+s_{j}(\bar{x})[\mathrm{cf}$. (12)], we obtain for the part without the fluctuations $s_{j}(\bar{x})$

$$
\hat{\mathcal{D}}^{\dagger} \hat{\mathcal{D}}\left(\left\{\sigma_{j}\right\}\right) \equiv \hat{\triangle}_{0}\left(\left\{\sigma_{j}\right\}\right)=\hat{\bar{P}} \cdot \hat{\bar{P}}+\hat{\mathcal{M}}_{0}^{\dagger} \hat{\mathcal{M}}_{0}
$$

where

$$
\hat{\mathcal{M}}_{0}^{\dagger} \hat{\mathcal{M}}_{0}=\frac{1}{2}\left[\begin{array}{ll}
{\left[2 \sigma_{0}^{2}+2 \sigma_{1}^{2}+\left(\sigma_{2}^{2}+\sigma_{3}^{2}\right)\left(1+\gamma_{5}\right)\right]} & {\left[-\left(\sigma_{0}-i \sigma_{1}\right)\left(\sigma_{2}+i \sigma_{3}\right)\left(1-\gamma_{5}\right)\right]} \\
{\left[-\left(\sigma_{0}+i \sigma_{1}\right)\left(\sigma_{2}-i \sigma_{3}\right)\left(1-\gamma_{5}\right)\right]} & {\left[\left(\sigma_{2}^{2}+\sigma_{3}^{2}\right)\left(1-\gamma_{5}\right)\right]}
\end{array}\right] .
$$

In order to calculate the various parts of the effective potential, in particular the leading- $N_{\mathrm{c}}$ part $N_{\mathrm{c}} V^{(0)}$ of (16), it is very convenient to work in the rotated basis in which the $8 \times 8$ matrix (A.2) becomes diagonal. There exist many unitary $8 \times 8$ matrices which accomplish such rotations. We will use the following one which has the convenient property that it goes to the identity matrix when the expectation values of the charged Goldstones go to zero $\left(\sigma_{2}\right.$ and $\left.\sigma_{3} \rightarrow 0\right)$ :

$$
U^{\dagger} \hat{\mathcal{M}}_{0}^{\dagger} \hat{\mathcal{M}}_{0} U=\sum_{j=0}^{3} \sigma_{j}^{2}\left[\begin{array}{ll}
1 & 0 \\
0 & 0
\end{array}\right]
$$

where the above $8 \times 8$ diagonal matrix is a block matrix made up of four blocks $(1,0)$ of dimension $4 \times 4$, and $U$ is the following unitary matrix:

$$
U=\left[\begin{array}{cc}
\left(k_{0}^{(+)}+\gamma_{5} k_{0}^{(-)}\right) & g_{0}^{(+)}\left(1-\gamma_{5}\right) \\
-g_{0}^{(-)}\left(1-\gamma_{5}\right) & \left(k_{0}^{(+)}+\gamma_{5} k_{0}^{(-)}\right)
\end{array}\right]
$$

Here we used the following notations:

$$
\begin{aligned}
& k_{0}^{( \pm)}=\frac{1}{2}\left[1 \pm \sqrt{\frac{\sigma_{0}^{2}+\sigma_{1}^{2}}{\sum_{j=0}^{3} \sigma_{j}^{2}}}\right] \\
& g_{0}^{( \pm)}=\frac{\left(\sigma_{2} \pm i \sigma_{3}\right)}{2 \sqrt{\sum_{j=0}^{3} \sigma_{j}^{2}}} e^{\mp i \delta_{0}}, \quad \text { where: } \delta_{0}=\arg \left(\sigma_{0}+i \sigma_{1}\right) .
\end{aligned}
$$

From $($ A.3 $)$ we see that the trace $\operatorname{Tr} \hat{\mathcal{D}}^{\dagger} \hat{\mathcal{D}}\left(\left\{\sigma_{j}\right\}\right)$, which is relevant for calculation of various parts of the effective potential, is a function of the $S U(2)_{L} \times U(1)_{Y}$ invariant combination $\sigma^{2}=\sum_{0}^{3} \sigma_{j}^{2}=g \Phi_{0}^{\dagger} \Phi_{0}$ 
of the scalar field expectation values. In Sec. II [cf. Eq. (17)], the use of (A.3) was crucial to derive the general formula $(18)$ for the leading- $N_{\mathrm{c}}$ part $N_{\mathrm{c}} V^{(0)}$ of the effective potential.

\section{Appendix B Explicit calculation of $V^{(0)}$ and $V^{(1)}$}

The general expression for the leading- $N_{\mathrm{c}}$ part $N_{\mathrm{c}} V^{(0)}$ in terms of $\sigma^{2}=g \Phi_{0}^{\dagger} \Phi_{0}$ is given in (18) and can be rewritten in terms of $\sigma^{2}$ and the four-fermion coupling $G$

$$
N_{\mathrm{c}} V^{(0)}\left(\sigma^{2} ; G\right)=\frac{\sigma^{2}}{G}+\frac{N_{\mathrm{c}}}{8 \pi^{2}} \int_{0}^{\infty} \frac{d \tau}{\tau^{3}} \rho_{\mathrm{f}}(\tau)\left(e^{-\tau \sigma^{2}}-1\right)
$$

Consequently, when using the dimensionless notation (35)-(37), we obtain for the leading- $N_{\mathrm{c}}$ part of the effective potential in the PTC case (20) the following expression:

$$
\begin{aligned}
& \Xi^{(0)}\left(\varepsilon^{2} ; a\right)^{(\mathrm{PTC})}=\frac{8 \pi^{2}}{\Lambda_{\mathrm{f}}^{4} N_{\mathrm{c}}} N_{\mathrm{c}} V^{(0)}\left(\sigma^{2}=\varepsilon^{2} \Lambda_{\mathrm{f}}^{2} ; G\right)^{(\mathrm{PTC})}=\frac{\varepsilon^{2}}{a}+\int_{1}^{\infty} \frac{d z}{z^{3}}\left(e^{-z \varepsilon^{2}}-1\right) \\
& =\frac{\varepsilon^{2}}{a}+\frac{\varepsilon^{4}}{2}\left[e^{-\varepsilon^{2}} \varepsilon^{-2}\left(\varepsilon^{-2}-1\right)-\operatorname{li}\left(e^{-\varepsilon^{2}}\right)-\varepsilon^{-4}\right]=\frac{1}{2}\left\{\varepsilon^{2} 2\left(\frac{1}{a}-1\right)\right. \\
& \left.\quad-\varepsilon^{4} \ln \left(\varepsilon^{2}\right)+\varepsilon^{4}\left(\frac{3}{2}-\mathcal{C}\right)+\varepsilon^{6}\left(\frac{2}{3 ! 1}\right)+\cdots+(-1)^{n+1} \varepsilon^{2 n}\left(\frac{2}{n !(n-2)}\right)+\cdots\right\} .
\end{aligned}
$$

In the above formula, $l i$ is the conventional Logarithm-integral function, for which we used the conventional expansion in powers of $\varepsilon^{2}$ (i.e., in inverse powers of $\Lambda_{\mathrm{f}}^{2}$ ); $\mathcal{C}$ appearing in this expansion is Euler's constant $(\mathcal{C}=0.577215 \ldots)$. The PV cutoff case (21) gives analogously

$$
\begin{aligned}
& \Xi^{(0)}\left(\varepsilon^{2} ; a\right)^{(\mathrm{PV})}=\frac{8 \pi^{2}}{\Lambda_{\mathrm{f}}^{4} N_{\mathrm{c}}} N_{\mathrm{c}} V^{(0)}\left(\sigma^{2}=\varepsilon^{2} \Lambda_{\mathrm{f}}^{2} ; G\right)^{(\mathrm{PV})}=\frac{\varepsilon^{2}}{a}+\int_{0}^{\infty} \frac{d z}{z^{3}}\left(1-e^{-z}\right)^{2}\left(e^{-z \varepsilon^{2}}-1\right) \\
& =\frac{\varepsilon^{2}}{a}+\lim _{w \rightarrow \infty} \int_{1 / w}^{\infty} \frac{d z}{z^{3}}\left(1-2 e^{-z}+e^{-2 z}\right)\left(e^{-z \varepsilon^{2}}-1\right)=\cdots \\
& =\left[\frac{\varepsilon^{2}}{a}-\frac{\varepsilon^{4}}{2} \ln \left(\varepsilon^{2}\right)+\left(1+\varepsilon^{2}\right)^{2} \ln \left(1+\varepsilon^{2}\right)-\frac{1}{2}\left(2+\varepsilon^{2}\right)^{2} \ln \left(2+\varepsilon^{2}\right)+2 \ln 2\right]
\end{aligned}
$$

From here we get the first derivative in the two regularization cases:

$$
\begin{aligned}
& {\frac{\partial \Xi^{(0)}\left(\varepsilon^{2} ; a\right)}{\partial \varepsilon^{2}}}^{(\mathrm{PTC})}=\frac{8 \pi^{2}}{\Lambda_{\mathrm{f}}^{2}} \frac{\partial}{\partial \sigma^{2}} V^{(0)}\left(\sigma^{2} \Lambda_{\mathrm{f}}^{2} \varepsilon^{2} ; G\right)^{(\mathrm{PTC})} \\
& \quad=\left\{\frac{1}{a}-1-\varepsilon^{2} \ln \left(\varepsilon^{2}\right)+\varepsilon^{2}(1-\mathcal{C})+\frac{\varepsilon^{4}}{2}-\frac{\varepsilon^{6}}{12}+\cdots+(-1)^{n} \frac{\varepsilon^{2 n}}{n !(n-1)}+\cdots\right\}, \\
& {\frac{\partial \Xi^{(0)}\left(\varepsilon^{2} ; a\right)}{\partial \varepsilon^{2}}}^{(\mathrm{PV})}=\left\{\frac{1}{a}-\varepsilon^{2} \ln \left(\varepsilon^{2}\right)+2\left(1+\varepsilon^{2}\right) \ln \left(1+\varepsilon^{2}\right)-\left(2+\varepsilon^{2}\right) \ln \left(2+\varepsilon^{2}\right)\right\} .
\end{aligned}
$$


These expressions are zero at the corresponding leading- $N_{\mathrm{c}}$ gap equation solutions $\varepsilon^{2}=\varepsilon_{0}^{2}$.

Now we turn to the calculation of the NTL part of the effective potential. The operators $\hat{\triangle}_{1}$ and $\hat{\triangle}_{2}$ of (22), which are linear and quadratic in the scalar fields fluctuations $\left\{s_{j}(\bar{x})\right\}$, respectively, are obtained from (A.1) by simply using in the $\hat{\mathcal{M}}=\hat{\mathcal{M}}_{0}+\delta \hat{\mathcal{M}}$ matrix of (乐) the full fluctuating values $\tilde{\sigma}_{j}(\bar{x})=\sigma_{j}+s_{j}(\bar{x})$, and extracting from there the terms linear and quadratic in $\left\{s_{j}(\bar{x})\right\}$

$$
\begin{aligned}
& \hat{\triangle}_{1}\left(\left\{\sigma_{j} ; s_{j}(\bar{x})\right\}\right)=\hat{\mathcal{M}}_{0}^{\dagger} \delta \hat{\mathcal{M}}+\delta \hat{\mathcal{M}}^{\dagger} \hat{\mathcal{M}}_{0}+i \bar{\gamma}_{\mu}\left(\frac{\partial}{\partial \bar{x}_{\mu}} \delta \hat{\mathcal{M}}\right) \\
& =\begin{array}{ll}
{\left[2 \sigma_{0} s_{0}+2 \sigma_{1} s_{1}+\left(\sigma_{2} s_{2}+\sigma_{3} s_{3}\right)\left(1+\gamma_{5}\right)\right]} & {\left[\left(-\sigma_{n}^{(-)} s_{c}^{(+)}-\sigma_{c}^{(+)} s_{n}^{(-)}\right)\left(1-\gamma_{5}\right)\right]} \\
{\left[\begin{array}{ll}
\left(-\sigma_{n}^{(+)} s_{c}^{(-)}-\sigma_{c}^{(-)} s_{n}^{(+)}\right)\left(1-\gamma_{5}\right) & {\left[\left(\sigma_{2} s_{2}+\sigma_{3} s_{3}\right)\left(1-\gamma_{5}\right)\right]}
\end{array}\right]}
\end{array} \\
& +i \bar{\gamma}_{\mu}\left[\begin{array}{lll}
{\left[\frac{\partial}{\partial \bar{x}_{\mu}}\left(s_{0}-i \gamma_{5} s_{1}\right)\right]} & {\left[-\frac{1}{\sqrt{2}} \frac{\partial}{\partial \bar{x}_{\mu}} s_{c}^{(+)}\left(1-\gamma_{5}\right)\right]} \\
{\left[\begin{array}{ll}
\left.-\frac{1}{\sqrt{2}} \frac{\partial}{\partial \bar{x}_{\mu}} s_{c}^{(-)}\left(1+\gamma_{5}\right)\right] & 0
\end{array}\right]}
\end{array}\right. \\
& \hat{\triangle}_{2}\left(\left\{\sigma_{j} ; s_{j}(\bar{x})\right\}\right)=\delta \hat{\mathcal{M}}^{\dagger} \delta \hat{\mathcal{M}} \\
& =\frac{1}{2}\left[\begin{array}{ll}
{\left[2 s_{0}^{2}+2 s_{1}^{2}+\left(s_{2}^{2}+s_{3}^{2}\right)\left(1+\gamma_{5}\right)\right]} & {\left[-2 s_{n}^{(-)} s_{c}^{(+)}\left(1-\gamma_{5}\right)\right]} \\
{\left[-2 s_{n}^{(+)} s_{c}^{(-)}\left(1-\gamma_{5}\right)\right]}
\end{array}\right]
\end{aligned}
$$

where we used shorthand notations

$$
\begin{array}{rlrl}
\sigma_{n}^{( \pm)} & =\frac{1}{\sqrt{2}}\left(\sigma_{0} \pm i \sigma_{1}\right), & s_{n}^{( \pm)}=\frac{1}{\sqrt{2}}\left(s_{0} \pm i s_{1}\right) \\
\sigma_{c}^{( \pm)}=\frac{1}{\sqrt{2}}\left(\sigma_{2} \pm i \sigma_{3}\right), & s_{c}^{( \pm)}=\frac{1}{\sqrt{2}}\left(s_{2} \pm i s_{3}\right) .
\end{array}
$$

We use the expressions (B.6)-(B.7) to calculate the proper time integrals of the bosonic effective action in the curly brackets of the exponent in the path integral of (23). The first question appearing at this point is: in which basis is it most convenient to calculate the traces over the 8-dimensional spinor/isospin degrees of freedom? It seems that the most convenient basis is the one given by (A.3)(A.4), in which the matrix $\hat{\triangle}_{0}$, and hence the matrix $\exp \left(-\tau_{j} \hat{\triangle}_{0}\right)$, are diagonal and explicitly known through (A.3). The matrices $\hat{\triangle}_{1}$ and $\hat{\triangle}_{2}$, on the other hand, are in this basis not diagonal, but can be calculated explicitly by help of $(\mathrm{A} .4)$ and (B.6)-(B.7). The tracing over the configuration space is most easily carried out by using for the $U$-rotated operators $U^{\dagger} \hat{\triangle}_{0} U$ the momentum basis, and for the $U$-rotated operators $U^{\dagger} \hat{\triangle}_{j} U(j=1,2)$ the coordinate basis

$$
\begin{gathered}
\left\langle\bar{q}^{\prime}\left|U^{\dagger} e^{-\tau_{j} \hat{\triangle}_{0}} U\right| \bar{q}\right\rangle=\delta\left(\bar{q}-\bar{q}^{\prime}\right) \exp \left(-\tau_{j} \bar{q}^{2}\right)\left[\begin{array}{cc}
\exp \left(-\tau_{j} \sum_{k=0}^{3} \sigma_{k}^{2}\right) & 0 \\
0 & 1
\end{array}\right], \\
\left\langle\bar{x}^{\prime}\left|U^{\dagger} \hat{\triangle}_{j} U\right| \bar{x}\right\rangle=\delta\left(\bar{x}-\bar{x}^{\prime}\right) U^{\dagger} \hat{\triangle}_{j}(\bar{x}) U
\end{gathered}
$$


In the above matrix, 1, 0 and $\exp (\cdots)$ represent the corresponding $4 \times 4$ blocks. First we perform the trivial integrations over those variables which appear in the $\delta$ functions. Subsequently, integrations by parts in the expression $\operatorname{Tr}\left[e^{-\tau_{1} \hat{\triangle}_{0}} \hat{\triangle}_{1} e^{-\tau_{2} \hat{\triangle}_{0}} \hat{\triangle}_{1}\right]$ result in the replacement of the partial derivatives in (B.6) by the corresponding (differences of) momenta. Integrations in the expression $\operatorname{Tr}\left[e^{-\tau \hat{\triangle}_{0}} \hat{\triangle}_{2}\right]$ are simpler. The tracing over the color degrees of freedom is trivial and gives factor $N_{\mathrm{c}}$, due to the $N_{\mathrm{c}} \times N_{\mathrm{c}}$ identity matrix structure of these operators in the color subspace. After longer, but straightforward algebraic manipulations along the lines outlined here, we obtain explicit integrals for the traces and end up with the following expression for the effective action $\Gamma$ in the curly brackets of the exponent of (23)

$$
\Gamma\left(\sigma^{2} ;\left\{v_{j}\right\} ;\left\{I_{j}\right\}\right)=-\frac{1}{2} \iint d^{4} \bar{x} d^{4} \bar{y} \sum_{j=0}^{3} v_{j}(\bar{y}) \hat{\mathcal{A}}_{j}\left(\bar{y}, \bar{x} ; \sigma^{2}\right) v_{j}(\bar{x})-i \sum_{j=0}^{3} I_{j} \int d^{4} \bar{x} v_{j}(\bar{x}),
$$

where $\sigma^{2}=\sum_{0}^{3} \sigma_{j}^{2}=g \Phi_{0}^{\dagger} \Phi_{0}$, and the kernels $\hat{\mathcal{A}}_{j}$ have the structure (29), with the terms there being the following explicit integrals over the proper time variables:

$$
\begin{aligned}
& \alpha^{(1)}\left(\sigma^{2} ; G\right)=\frac{2}{G}-\frac{N_{\mathrm{c}}}{4 \pi^{2}} \int_{0}^{\infty} \frac{d \tau}{\tau^{2}} \rho_{\mathrm{f}}(\tau) e^{-\tau \sigma^{2}}, \\
& \alpha^{(2)}\left(\sigma^{2}\right)=-\frac{N_{\mathrm{c}}}{8 \pi^{2}} \int_{0}^{\infty} \frac{d \tau}{\tau^{2}} \rho_{\mathrm{f}}(\tau)\left(1-e^{-\tau \sigma^{2}}\right), \\
& \left(\beta_{1}^{(1)} \pm \beta_{2}^{(1)}\right)\left(\bar{x} ; \sigma^{2}\right)=\frac{N_{\mathrm{c}}}{8(2 \pi)^{4}} \int_{0}^{\infty} \int_{0}^{\infty} \frac{d \tau_{1} d \tau_{2}}{\tau_{1}^{2} \tau_{2}^{2}} \rho_{\mathrm{f}}\left(\tau_{1}+\tau_{2}\right) \exp \left[-\left(\tau_{1}+\tau_{2}\right) \sigma^{2}\right] \times \\
& \exp \left[-\frac{\left(\tau_{1}+\tau_{2}\right)}{4 \tau_{1} \tau_{2}} \bar{x}^{2}\right]\left[\frac{\left(\tau_{1}+\tau_{2}\right)}{2 \tau_{1} \tau_{2}}\left(4-\frac{\left(\tau_{1}+\tau_{2}\right)}{2 \tau_{1} \tau_{2}} \bar{x}^{2}\right)+2 \sigma^{2}(1 \pm 1)\right], \\
& \beta^{(2)}\left(\bar{x} ; \sigma^{2}\right)=\frac{N_{\mathrm{c}}}{16(2 \pi)^{4}} \int_{0}^{\infty} \int_{0}^{\infty} \frac{d \tau_{1} d \tau_{2}}{\tau_{1}^{2} \tau_{2}^{2}} \rho_{\mathrm{f}}\left(\tau_{1}+\tau_{2}\right) \exp \left[-\tau_{1} \sigma^{2}\right] \times \\
& \exp \left[-\frac{\left(\tau_{1}+\tau_{2}\right)}{4 \tau_{1} \tau_{2}} \bar{x}^{2}\right]\left[\frac{\left(\tau_{1}+\tau_{2}\right)}{1 \tau_{1} \tau_{2}}\left(4-\frac{\left(\tau_{1}+\tau_{2}\right)}{2 \tau_{1} \tau_{2}} \bar{x}^{2}\right)+2 \sigma^{2}\right] \text {. }
\end{aligned}
$$

The scalar field fluctuations $\left\{v_{j}(\bar{x})\right\}$ are specific orthonormal combinations of the original scalar field fluctuations $\left\{s_{j}(\bar{x})\right\}$

$$
v_{j}(\bar{x})=\mathcal{O}_{j k} s_{k}(\bar{x}),
$$

where $\mathcal{O}$ is the following orthonormal $4 \times 4$ matrix:

$$
\mathcal{O}=\frac{1}{\sqrt{\sum_{k=0}^{3} \sigma_{k}^{2}}}\left[\begin{array}{llll}
\sigma_{0} & \sigma_{1} & \sigma_{2} & \sigma_{3} \\
-\sigma_{1} & \sigma_{0} & -\sigma_{3} & \sigma_{2} \\
\varsigma^{-1} \sigma_{0} & \varsigma^{-1} \sigma_{1} & -\varsigma \sigma_{2} & -\varsigma \sigma_{3} \\
-\varsigma^{-1} \sigma_{1} & \varsigma^{-1} \sigma_{0} & \varsigma \sigma_{3} & -\varsigma \sigma_{2}
\end{array}\right]
$$


and we denoted here:

$$
\varsigma=\sqrt{\frac{\sigma_{0}^{2}+\sigma_{1}^{2}}{\sigma_{2}^{2}+\sigma_{3}^{2}}} .
$$

In the special case of our interest, only the neutral scalar (Higgs) component acquires nonzero value, i.e., $\sigma_{1}=\sigma_{2}=\sigma_{3}=0$. In this limiting case (we have the freedom to additionally require: $\sigma_{3} / \sigma_{2} \rightarrow 0$ ), the fluctuations $\left\{v_{j}(\bar{x})\right\}$ reduce to the old fluctuations of the non-rotated fields: $v_{0}=s_{0}, v_{1}=s_{1}$, $v_{2}=-s_{2}, v_{3}=-s_{3}$, and the scalar expectation value $\sigma$ reduces to $\sigma_{0}$.

Comparing ( $\mathrm{B} .1)$ and $(\mathrm{B} .10)$, we see immediately that $\alpha^{(1)}\left(\sigma^{2} ; G\right)$ is exactly twice the derivative with respect to $\sigma^{2}$ of the leading- $N_{\mathrm{c}}$ part of the effective potential $N_{\mathrm{c}} V^{(0)}\left(\sigma^{2} ; G\right)=\Lambda_{\mathrm{f}}^{4} N_{\mathrm{c}} \Xi^{(0)} /\left(8 \pi^{2}\right)$, i.e., relation (31). In the PTC case (20), the relation (B.10) gives

$$
\begin{aligned}
\alpha^{(1)}\left(\sigma^{2} ; G\right)^{(\mathrm{PTC})=} & \frac{2}{G}-\frac{N_{\mathrm{c}}}{4 \pi^{2}}\left\{\Lambda_{\mathrm{f}}^{2}-\sigma^{2} \ln \left(\frac{\Lambda_{\mathrm{f}}^{2}}{\sigma^{2}}\right)-(1-\mathcal{C}) \sigma^{2}-\frac{1}{2} \frac{\sigma^{4}}{\Lambda_{\mathrm{f}}^{2}}\right. \\
& \left.+\frac{1}{12} \frac{\sigma^{6}}{\Lambda_{\mathrm{f}}^{4}}+\cdots+(-1)^{n+1} \frac{1}{n !(n-1)} \frac{\sigma^{2 n}}{\Lambda_{\mathrm{f}}^{2 n-2}}+\cdots\right\},
\end{aligned}
$$

which is compatible with (B.4). The Fourier transformed quantities $\tilde{\beta}_{j}^{(i)}\left(\bar{p}^{2} ; \sigma^{2}\right)$, appearing in the NTL part (30), can be calculated from (B.11) by first carrying out explicitly the integration over $d^{4} \bar{x}$

$$
\begin{aligned}
\left(\tilde{\beta}_{1}^{(1)} \pm \tilde{\beta}_{2}^{(1)}\right)\left(\bar{p}^{2} ; \sigma^{2}\right)= & \frac{N_{\mathrm{c}}}{8 \pi^{2}}\left[\bar{p}^{2}+2(1 \pm 1) \sigma^{2}\right] \int_{0}^{\infty} \int_{0}^{\infty} \frac{d \tau_{1} d \tau_{2}}{\left(\tau_{1}+\tau_{2}\right)^{2}} \rho_{\mathrm{f}}\left(\tau_{1}+\tau_{2}\right) \exp \left[-\left(\tau_{1}+\tau_{2}\right) \sigma^{2}\right] \\
& \times \exp \left[-\frac{\tau_{1} \tau_{2} \bar{p}^{2}}{\left(\tau_{1}+\tau_{2}\right)}\right] \\
\alpha^{(2)}\left(\sigma^{2}\right)+\tilde{\beta}^{(2)}\left(\bar{p}^{2} ; \sigma^{2}\right)= & \frac{N_{\mathrm{c}}}{4 \pi^{2}} \bar{p}^{2} \int_{0}^{\infty} \int_{0}^{\infty} \frac{d \tau_{1} d \tau_{2}}{\left(\tau_{1}+\tau_{2}\right)^{3}} \tau_{1} \rho_{\mathrm{f}}\left(\tau_{1}+\tau_{2}\right) \exp \left(-\tau_{1} \sigma^{2}\right) \exp \left[-\frac{\tau_{1} \tau_{2} \bar{p}^{2}}{\left(\tau_{1}+\tau_{2}\right)}\right] .
\end{aligned}
$$

In the last formula, after the Fourier transformation we performed the substitution $\tau=\tau_{1}+\tau_{2}$ and carried out integration by parts over $d \tau_{1}$, and then reintroduced $\tau_{2}$.

We now introduce the new variables $z$ and $\tau: \tau_{1}=\tau z, \tau_{2}=\tau(1-z)$, where $\tau$ and $z$ run through the intervals $[0, \infty]$ and $[0,1]$, respectively. The above quantities $(\mathrm{B} .15)$ and $(\overline{B .16})$ can then be written in the form

$$
\begin{gathered}
\left(\tilde{\beta}_{1}^{(1)} \pm \tilde{\beta}_{2}^{(1)}\right)\left(\bar{p}^{2} ; \sigma^{2}\right)=\frac{N_{\mathrm{c}}}{8 \pi^{2}}\left[\bar{p}^{2}+2(1 \pm 1) \sigma^{2}\right] \int_{0}^{1} d z \int_{0}^{\infty} \frac{d \tau}{\tau} \rho_{\mathrm{f}}(\tau) \exp \left\{-\tau\left[\bar{p}^{2} z(1-z)+\sigma^{2}\right]\right\} \\
\alpha^{(2)}\left(\sigma^{2}\right)+\tilde{\beta}^{(2)}\left(\bar{p}^{2} ; \sigma^{2}\right)=\frac{N_{\mathrm{c}}}{4 \pi^{2}} \bar{p}^{2} \int_{0}^{1} d z z \int_{0}^{\infty} \frac{d \tau}{\tau} \rho_{\mathrm{f}}(\tau) \exp \left\{-\tau\left[\bar{p}^{2} z(1-z)+\sigma^{2} z\right]\right\}
\end{gathered}
$$


In the PTC case, the integration over $d \tau$ gives us the Logarithm-integral

$$
\begin{aligned}
\int_{1 / \Lambda_{\mathrm{f}}^{2}}^{\infty} \frac{d \tau}{\tau} \exp \left[-\tau \mathcal{F}\left(z ; \bar{p}^{2}, \sigma^{2}\right)\right] & =-\operatorname{li}\left(e^{-\mathcal{F} / \Lambda_{\mathrm{f}}^{2}}\right) \\
& =-\left.\left[\mathcal{C}+\ln x+\sum_{k=1}^{\infty}(-1)^{k} \frac{x^{k}}{k ! k}\right]\right|_{x=\mathcal{F}\left(z ; \bar{p}^{2}, \sigma^{2}\right) / \Lambda_{\mathrm{f}}^{2}},
\end{aligned}
$$

where $\mathcal{C}$ is Euler's constant $(\mathcal{C}=0.577215 \ldots)$. The integration over $d z$ can then be carried out term by term, and we end up with the following series in inverse powers of the cutoff $\Lambda_{\mathrm{f}}$ for the PTC case:

$$
\begin{aligned}
& \frac{8 \pi^{2}}{N_{\mathrm{c}}} \frac{\left(\tilde{\beta}_{1}^{(1)} \pm \tilde{\beta}_{2}^{(1)}\right)\left(\bar{p}^{2} ; \sigma^{2}\right)^{(\mathrm{PTC})}}{\left[\bar{p}^{2}+2 \sigma^{2}(1 \pm 1)\right]}=\left\{\ln \left(\frac{\Lambda_{\mathrm{f}}^{2}}{\sigma^{2}}\right)+\left[-\mathcal{C}-\left.\frac{2}{3} z \mathrm{~F}(z)\right|_{z=\bar{p}^{2} /\left(\bar{p}^{2}+4 \sigma^{2}\right)}\right]\right. \\
& +\left[\frac{\sigma^{2}}{\Lambda_{\mathrm{f}}^{2}}+\frac{1}{6} \frac{\bar{p}^{2}}{\Lambda_{\mathrm{f}}^{2}}\right]-\frac{1}{4}\left[\left(\frac{\sigma^{2}}{\Lambda_{\mathrm{f}}^{2}}\right)^{2}+\frac{1}{3} \frac{\sigma^{2} \bar{p}^{2}}{\Lambda_{\mathrm{f}}^{4}}+\frac{1}{30}\left(\frac{\bar{p}^{2}}{\Lambda_{\mathrm{f}}^{2}}\right)^{2}\right]+\frac{1}{18}\left[\left(\frac{\sigma^{2}}{\Lambda_{\mathrm{f}}^{2}}\right)^{3}+\frac{1}{2} \frac{\sigma^{4} \bar{p}^{2}}{\Lambda_{\mathrm{f}}^{6}}\right. \\
& \left.\left.\quad+\frac{1}{10} \frac{\sigma^{2}\left(\bar{p}^{2}\right)^{2}}{\Lambda_{\mathrm{f}}^{6}}+\frac{1}{140}\left(\frac{\bar{p}^{2}}{\Lambda_{\mathrm{f}}^{2}}\right)^{3}\right]+(-2) \sum_{k=4}^{\infty} \frac{(-1)^{k}}{k ! k}\left(\frac{\bar{p}^{2}}{\Lambda_{\mathrm{f}}^{2}}\right)^{k} \int_{0}^{1 / 2} d u\left(\frac{\sigma^{2}}{\bar{p}^{2}}+\frac{1}{4}-u^{2}\right)^{k}\right\},(\mathrm{B} .20) \\
& \frac{8 \pi^{2}}{N_{\mathrm{c}} \bar{p}^{2}}\left[\alpha{ }^{(2)}\left(\sigma^{2}\right)+\tilde{\beta}^{(2)}\left(\bar{p}^{2} ; \sigma^{2}\right)\right]^{(\mathrm{PTC})}=\left\{\ln \left(\frac{\Lambda_{\mathrm{f}}^{2}}{\sigma^{2}}\right)+\left[-\mathcal{C}+2+\frac{\sigma^{2}}{\bar{p}^{2}}-\left(1+\frac{\sigma^{2}}{\bar{p}^{2}}\right)^{2} \ln \left(1+\frac{\bar{p}^{2}}{\sigma^{2}}\right)\right]\right. \\
& \quad+\left[\frac{2}{3} \frac{\sigma^{2}}{\Lambda_{\mathrm{f}}^{2}}+\frac{1}{6} \frac{\bar{p}^{2}}{\Lambda_{\mathrm{f}}^{2}}\right]-\frac{1}{4}\left[\frac{1}{2}\left(\frac{\sigma^{2}}{\Lambda_{\mathrm{f}}^{2}}\right)^{2}+\frac{1}{5} \frac{\sigma^{2} \bar{p}^{2}}{\Lambda_{\mathrm{f}}^{4}}+\frac{1}{30}\left(\frac{\bar{p}^{2}}{\Lambda_{\mathrm{f}}^{2}}\right)^{2}\right]+\frac{1}{45}\left[\left(\frac{\sigma^{2}}{\Lambda_{\mathrm{f}}^{2}}\right)^{3}+\frac{1}{2} \frac{\sigma^{4} \bar{p}^{2}}{\Lambda_{\mathrm{f}}^{6}}\right. \\
& \left.\left.+\frac{1}{7} \frac{\sigma^{2}\left(\bar{p}^{2}\right)^{2}}{\Lambda_{\mathrm{f}}^{6}}+\frac{1}{56}\left(\frac{\bar{p}^{2}}{\Lambda_{\mathrm{f}}^{2}}\right)^{3}\right]-2 \sum_{k=4}^{\infty} \frac{1}{k ! k}\left(\frac{\bar{p}^{2}}{\Lambda_{\mathrm{f}}^{2}}\right)^{k} \int_{0}^{1} d u u^{k+1}\left(u-1-\frac{\sigma^{2}}{\bar{p}^{2}}\right)^{k}\right\}^{(\mathrm{B} .21)}
\end{aligned}
$$

The explicit expression for the function $\mathrm{F}(z)$ appearing in the $\Lambda_{\mathrm{f}}$-independent part of $(\mathbb{B} .20)$ is

$$
\mathrm{F}(z)=\frac{3}{2} z^{-3 / 2} \ln \left(\frac{1+\sqrt{z}}{1-\sqrt{z}}\right)-\frac{3}{z}=1+\frac{3}{5} z+\frac{3}{7} z^{2}+\frac{3}{9} z^{3}+\cdots .
$$

For the PV cutoff case (21), we obtain the corresponding results, as given in (33) and (34), by very similar procedure: first we carry out integration over $d \tau$ in (B.17)-(B.18) according to (B.19), where we set $1 / \Lambda^{2}$ for the lower bound of integration; then we take the limit $\Lambda^{2} \rightarrow \infty$; the resulting integrands (sums of logarithms) are finite, and integration over $d z$ can be performed analytically; expansion of the results in inverse powers of $\Lambda_{\mathrm{f}}^{2}$ gives then the expressions given in (33) and (34). It is also possible to follow this procedure without expanding in inverse powers of $\Lambda_{\mathrm{f}}^{2}$. We then obtain, for $\bar{p}^{2}>0$, the following solutions for $\left(\tilde{\beta}_{1}^{(1)} \pm \tilde{\beta}_{2}^{(1)}\right)$ and $\left(\alpha^{(2)}+\tilde{\beta}^{(2)}\right)$ in the PV case:

$$
\frac{8 \pi^{2}}{N_{\mathrm{c}}} \frac{\left(\tilde{\beta}_{1}^{(1)} \pm \tilde{\beta}_{2}^{(1)}\right)\left(\bar{p}^{2} ; \sigma^{2}\right)^{(\mathrm{PV})}}{\left[\bar{p}^{2}+2 \sigma^{2}(1 \pm 1)\right]}=-\sum_{j=0}^{2} a_{j}\left[\left(1-2 \delta_{j}\right) \ln \left(\frac{\sigma^{2}+\lambda_{j}^{2}}{\bar{p}^{2}}\right)+4 \delta_{j} \ln \left(\delta_{j}+\frac{1}{2}\right)\right]
$$




$$
\begin{aligned}
& \frac{8 \pi^{2}}{N_{\mathrm{c}} \bar{p}^{2}}\left[\alpha^{(2)}\left(\sigma^{2}\right)+\tilde{\beta}^{(2)}\left(\bar{p}^{2} ; \sigma^{2}\right)\right]^{(\mathrm{PV})}=\left\{-\left(1+\frac{\sigma^{2}}{\bar{p}^{2}}\right)^{2} \ln \left(1+\frac{\bar{p}^{2}}{\sigma^{2}}\right)\right. \\
& \quad-\sum_{j=0}^{2} a_{j} \ln \left(\frac{\sigma^{2}+\lambda_{j}^{2}}{\bar{p}^{2}}\right)+\sum_{j=1}^{2} a_{j}\left[\frac{1}{2}\left(1+\frac{\sigma^{2}}{\bar{p}^{2}}\right)^{2}+\frac{\lambda_{j}^{2}}{\bar{p}^{2}}+\left(1+\frac{\sigma^{2}}{\bar{p}^{2}}\right) \eta_{j}\right] \ln \left(1+\frac{\sigma^{2}}{\lambda_{j}^{2}}\right) \\
& \left.\quad-2\left(1+\frac{\sigma^{2}}{\bar{p}^{2}}\right) \sum_{j=1}^{2} a_{j} \eta_{j}\left[\ln \left(\eta_{j}-\frac{\sigma^{2}}{2 \bar{p}^{2}}+\frac{1}{2}\right)-\ln \left(\eta_{j}-\frac{\sigma^{2}}{2 \bar{p}^{2}}-\frac{1}{2}\right)\right]\right\},
\end{aligned}
$$

where we use the notation

$$
\begin{array}{cc}
a_{0}=a_{1}=1, a_{2}=-2 ; & \lambda_{0}^{2}=0, \lambda_{1}^{2}=2 \Lambda_{\mathrm{f}}^{2}, \lambda_{2}^{2}=\Lambda_{\mathrm{f}}^{2} \\
\delta_{j}=\sqrt{\frac{\sigma^{2}+\lambda_{j}^{2}}{\bar{p}^{2}}+\frac{1}{4}} ; \quad \eta_{j}=\sqrt{\frac{1}{4}\left(1+\frac{\sigma^{2}}{\bar{p}^{2}}\right)^{2}+\frac{\lambda_{j}^{2}}{\bar{p}^{2}}} .
\end{array}
$$

\section{References}

[1] Y. NAmBU, in "New Theories in Physics", Proceedings of the XI International Symposium on Elementary Particle Physics, Kazimierz, Poland, 1988 (Z. Ajduk, S. Pokorski, and A. Trautman, Eds.), pp. 1-10, World Scientific, Singapore, 1989.

[2] V. A. Miransky, M. Tanabashi and K. Yamawaki, Mod. Phys. Lett. A 4 (1989), 1043; Phys. Lett. B 221 (1989), 177.

[3] W. J. Marciano, Phys. Rev. Lett. 62 (1989), 2793; Phys. Rev. D 41 (1990), 219.

[4] W. A. Bardeen, C. T. Hill And M. Lindner, Phys. Rev. D 41 (1990), 1647.

[5] S. F. King and S. H. Mannan, Phys. Lett. B 241 (1990), 249; Z. Phys. C 52 (1991), 59.

[6] M. Bando, T. Kugo, N. Maekawa, N. Sasakura and Y. Watabiki, Phys. Lett. B 246 (1990), 466; F. Cooper and J. Pérez-Mercader, Phys. Rev. D 43 (1991), 4129; J. Fröhlich and L. Lavoura, Phys. Lett. B 253 (1991), 218; P. Fishbane, R. E. Norton and T. N. Truong, Phys. Rev. D 46 (1992), 1768; P. Fishbane And R. E. Norton, Phys. Rev. D 48 (1993), 4924.

[7] Y.-B. Dai, Y.-B. Ding, C.-S. Huang And C.-L. Wang, Phys. Lett. B 285 (1992), 245; K.-I. Kondo, M. Tanabashi And K. Yamawaki, Prog. Theor. Phys. 89 (1993), 1299; A. Blumhofer, R. Dawid And M. Lindner, Phys. Lett. B $\mathbf{3 6 0}$ (1995), 123.

[8] J. Zinn-Justin, Nucl. Phys. B 367 (1991), 105;

[9] A. Blumhofer, Phys. Lett. B 230 (1994), 352; Nucl. Phys. B 437 (1995), 25.

[10] C. T. Hill, M. A. Luty and E. A. Paschos, Phys. Rev. D 43 (1991), 3011; S. P. Martin, Phys. Rev. D 44 (1991), 2892; Y. Achiman And A. Davidson, Phys. Lett. B 261 (1991), 431. 
[11] M. A. Luty, Phys. Rev. D 41 (1990), 2893; M. Suzuki, Phys. Rev. D 41 (1990), 3457; M. Harada And N. Kitazawa, Phys. Lett. B 257 (1991), 383; S. F. King And M. Suzuki, Phys. Lett. B 277 (1992), 153.

[12] T. K. Kuo, U. Mahanta And G. T. Park, Phys. Lett. B 248 (1990), 119; R. Bönisch, Phys. Lett. B 268 (1991), 394; K. S. Babu And R. N. Mohapatra, Phys. Rev. Lett. 66 (1991), 556; S. F. King, Phys. Rev. D 45 (1992), 990; S. P. Martin, Phys. Rev. D 45 (1992), 4283; ibid 46 (1992), 2197; G. P. Arata And K. Konishi, Phys. Lett. B 278 (1992), 351; M. A. Luty, Phys. Rev. D 48 (1993), 1295; M. Lindner And D. Ross, Nucl. Phys. B 370 (1992), 30; M. Lindner, Int. J. Mod. Phys. A 8 (1993), 2167 and references therein; A. Kundu, S. Raychaudhuri, T. De And B. Dutta-Roy, Phys. Rev. D 50 (1994), 6872; D. E. Kahana And S. H. Kahana, Phys. Rev. D 43 (1991), 2361; ibid 52 (1995), 3065; E. Akhmedov, M. Lindner, E. Schnapka And J. W. F. VAlle, Phys. Rev. D 53 (1996), 2752; Y. Yoshida, Phys. Rev. D 53 (1996), 7306.

[13] M. Carena, T. E. Clark, C. E. M. Wagner, W. A. Bardeen and K. Sasaki, Nucl. Phys. B 369 (1992), 33; W. A. Bardeen, M. Carena, S. Pokorski and C. E. M. Wagner, Phys. Lett. B 320 (1994), 110.

[14] L. Lavoura, Int. J. Mod. Phys. A 7 (1992), 3593; W. T. A. Ter Veldhuis, Phys. Rev. D 45 (1992), 3201.

[15] F. A. Barrios and U. Mahanta, Phys. Rev. D 43 (1991), 284.

[16] K. YAMAWAKI, hep-ph/9603293, to appear in "Dynamical Symmetry Breaking and Effective Field Theory", Proceedings of the 14th Symposium on Theoretical Physics, Cheju, Korea, July 21-26, 1995.

[17] Y. Nambu and G. Jona-Lasinio, Phys. Rev. 122 (1961), 345.

[18] V. G. Vaks And A. I. Larkin, J. Exp. Theor. Phys. 13 (1961), 192.

[19] G. Cvetič, E. A. Paschos and N. D. Vlachos, Phys. Rev. D 53 (1996), 2820.

[20] G. Cvetič And N. D. Vlachos, Phys. Lett. B 377 (1996), 102.

[21] R. S. Willey, Phys. Rev. D 48 (1993), 2877.

[22] T. Gherghetta, Phys. Rev. D 50 (1994), 5985.

[23] T. Kugo, Prog. Theor. Phys. 55 (1976), 2032; K. Kikkawa, ibid. 56 (1976), 947.

[24] S. WeinBerg, Phys. Rev. D 7 (1973), 2887.

[25] K. Higashijima, Prog. Theor. Phys. Suppl. 104 (1991).

[26] R. D. BALl, Phys. Rep. Vol. 182, No. 1 (1989).

[27] G. CvetiČ And E. A. Paschos, Nucl. Phys. B 395 (1993), 581.

[28] E. N. Nikolov, W. Broniowski, C. V. Christov, G. Ripka and K. Goeke, Bochum Univ. preprint RUB-TPII-13/95, hep-ph/9602274. 
[29] F. ABE et al. (CDF), Phys. Rev. Lett. 74 (1995), 2626; S. ADACHI et al. (D0), Phys. Rev. Lett. 74 (1995), 2632.

[30] H. Pagels and S. Stokar, Phys. Rev. D 20 (1979), 2947.

[31] J. M. Cornwall, R. Jackiw and E. Tomboulis, Phys. Rev. D 10 (1974), 2428.

[32] A. Hasenfratz, P. Hasenfratz, K. Jansen, J. Kuti And Y. Shen, Nucl. Phys. B 365 (1991), 79;

[33] M. Suzuki, Mod. Phys. Lett. A, 5 (1990), 1205; S. F. King And S. H. Mannan, Int. J. Mod. Phys. A 6 (1991), 4949.

[34] C. T. HiLl, Phys. Lett. B 266 (1991), 419.

[35] V. Dmitrašinović, H.-J. Schulze, R. Tegen And R. H. Lemmer, Ann. Phys. (N.Y.) 238 (1995), 332.

[36] S. Hands, A. Kocić And J. B. Kogut, Phys. Lett. B 273 (1991), 111; Ann. Phys. (N.Y.) 224 (1993), 29; J. A. Gracey, Phys. Lett. B 308 (1993), 65; Z. Phys. C 61 (1994), 115; S. E. Derkachov, N. A. Kivel, A. S. StePanenko And A. N. VASIL'Ev, in "Hadrons-93", Conference proceedings, Novy Svit, 1993; Saclay preprint SACLAY-SPHT-93-016, hep-th/9302034.

[37] K. Akama, Phys. Rev. Lett. 76 (1996), 184.

[38] D. Lurié And G. B. Tupper, Phys. Rev. D 47 (1993), 3580. 
Table 1

\begin{tabular}{|c|l|l||c||c|c||c|c||c|c|}
\hline$a$ & $\Lambda_{\mathrm{b}} / \Lambda_{\mathrm{f}}$ & $\frac{m_{t}(\Lambda)}{m_{t}^{(0)}}$ & $\frac{m_{t}^{\mathrm{ren}}}{m_{t}^{(0)}}$ & $\frac{m_{t}(\Lambda)}{m_{t}^{(0)}}$ & $\frac{m_{t}^{\mathrm{ren}}}{m_{t}^{(0)}}$ & $\frac{m_{t}(\Lambda)}{m_{t}^{(0)}}$ & $\frac{m_{t}^{\mathrm{ren}}}{m_{t}^{(0)}}$ \\
$(\mathrm{PTC})$ & $(\mathrm{PV})$ & $\Lambda_{\mathrm{b}}$ & $\begin{array}{c}\Lambda_{\mathrm{f}}^{(\mathrm{PTC})} \\
(\mathrm{PV})\end{array}$ & $(\mathrm{S})$ & $(\mathrm{S})$ & {$[\mathrm{TeV}]$} & {$[\mathrm{TeV}]$} \\
\hline \hline 1.273 & $1 / 2$ & $0.500(=\sqrt{1 / 4})$ & 0.475 & 0.481 & 0.445 & 0.517 & 0.488 & 0.724 & 1.448 \\
1.314 & $1 / 2$ & $0.577(=\sqrt{1 / 3})$ & 0.549 & 0.555 & 0.513 & 0.594 & 0.559 & 0.581 & 1.161 \\
1.458 & $1 / 2$ & $0.707(=\sqrt{1 / 2})$ & 0.669 & 0.678 & 0.623 & 0.720 & 0.673 & 0.387 & 0.773 \\
1.982 & $1 / 2$ & $0.816(=\sqrt{2 / 3})$ & 0.760 & 0.775 & 0.699 & 0.824 & 0.757 & 0.231 & 0.462 \\
\hline 1.665 & $1 / \sqrt{2}$ & $0.500(=\sqrt{1 / 4})$ & 0.453 & 0.473 & 0.407 & 0.531 & 0.470 & 0.663 & 0.938 \\
1.815 & $1 / \sqrt{2}$ & $0.577(=\sqrt{1 / 3})$ & 0.523 & 0.542 & 0.465 & 0.606 & 0.533 & 0.520 & 0.735 \\
2.736 & $1 / \sqrt{2}$ & $0.707(=\sqrt{1 / 2})$ & 0.630 & 0.641 & 0.532 & 0.724 & 0.618 & 0.306 & 0.433 \\
5.091 & $1 / \sqrt{2}$ & $0.738(=\sqrt{0.545})$ & 0.643 & 0.627 & 0.489 & 0.739 & 0.603 & 0.219 & 0.310 \\
\hline 3.613 & 1 & $0.500(=\sqrt{1 / 4})$ & 0.410 & 0.437 & 0.310 & 0.563 & 0.395 & 0.568 & 0.568 \\
7.935 & 1 & $0.560(=\sqrt{0.314})$ & 0.443 & 0.421 & 0.264 & 0.582 & 0.348 & 0.385 & 0.385 \\
\hline
\end{tabular}

Table 1: The bosonic cutoffs $\Lambda_{\mathrm{b}}$ and the quark (fermion) cutoff parameters $\Lambda_{\mathrm{f}}$, which result when we impose the requirement that the ratio $\varepsilon^{2} / \varepsilon_{0}^{2}=\left(m_{t}(\Lambda) / m_{t}^{(0)}\right)^{2}$ of the solution of the NTL gap equation with the solution of the leading- $N_{\mathrm{C}}$ quark loop gap equation not be smaller than: $1 / 4,1 / 3,1 / 2,2 / 3$, for the proper time cutoff (PTC) case. The corresponding ratios of the NTL-renormalized mass with the leading- $N_{\mathrm{C}}$ mass are also given. In addition, these mass ratios are given also for the corresponding cases [i.e., with the same $\Lambda_{\mathrm{b}}$ and the same four-fermion coupling $G$ of (11)] when the Pauli-Villars (PV) regulator and the simple covariant spherical (S) cutoff are applied to the fermionic momenta. We took $m_{t}^{\text {ren. }}=180 \mathrm{GeV}$. 


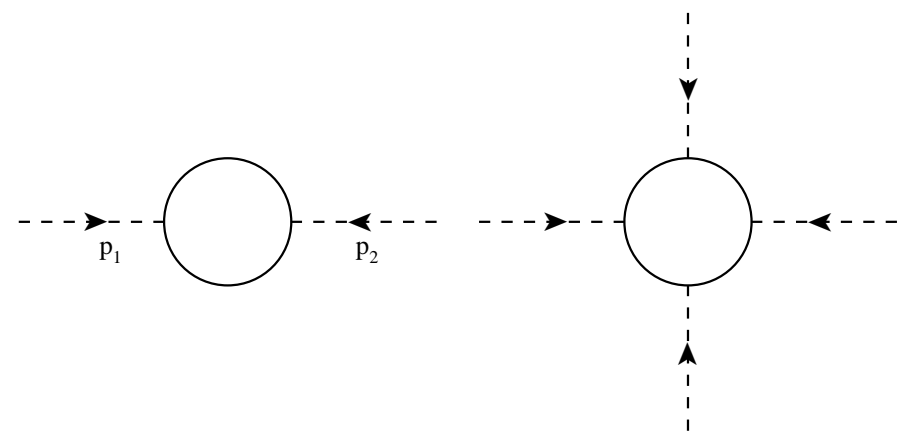

Fig. 1a

Fig. 1b

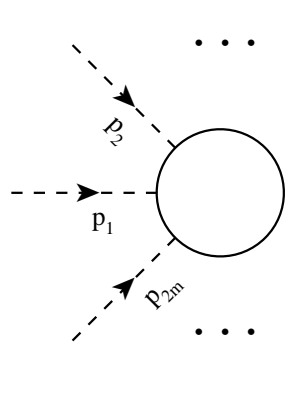

Fig. 1c

Figs. 1(a)-(c): The 1-loop 1-PI diagrams contributing to 1-PI Green functions $\tilde{\Gamma}_{H}^{(2 m ; 1)}\left(p_{1}, \ldots, p_{2 m}\right)$, which in turn yield the leading- $N_{\mathrm{C}}$ part $N_{\mathrm{C}} V^{(0)}$ in $1 / N_{\mathrm{C}}$ expansion of $V_{\mathrm{eff}}$. Full lines represent massless top quarks, and dotted external lines the scalar nondynamical Higgs of the Lagrangian (3). 


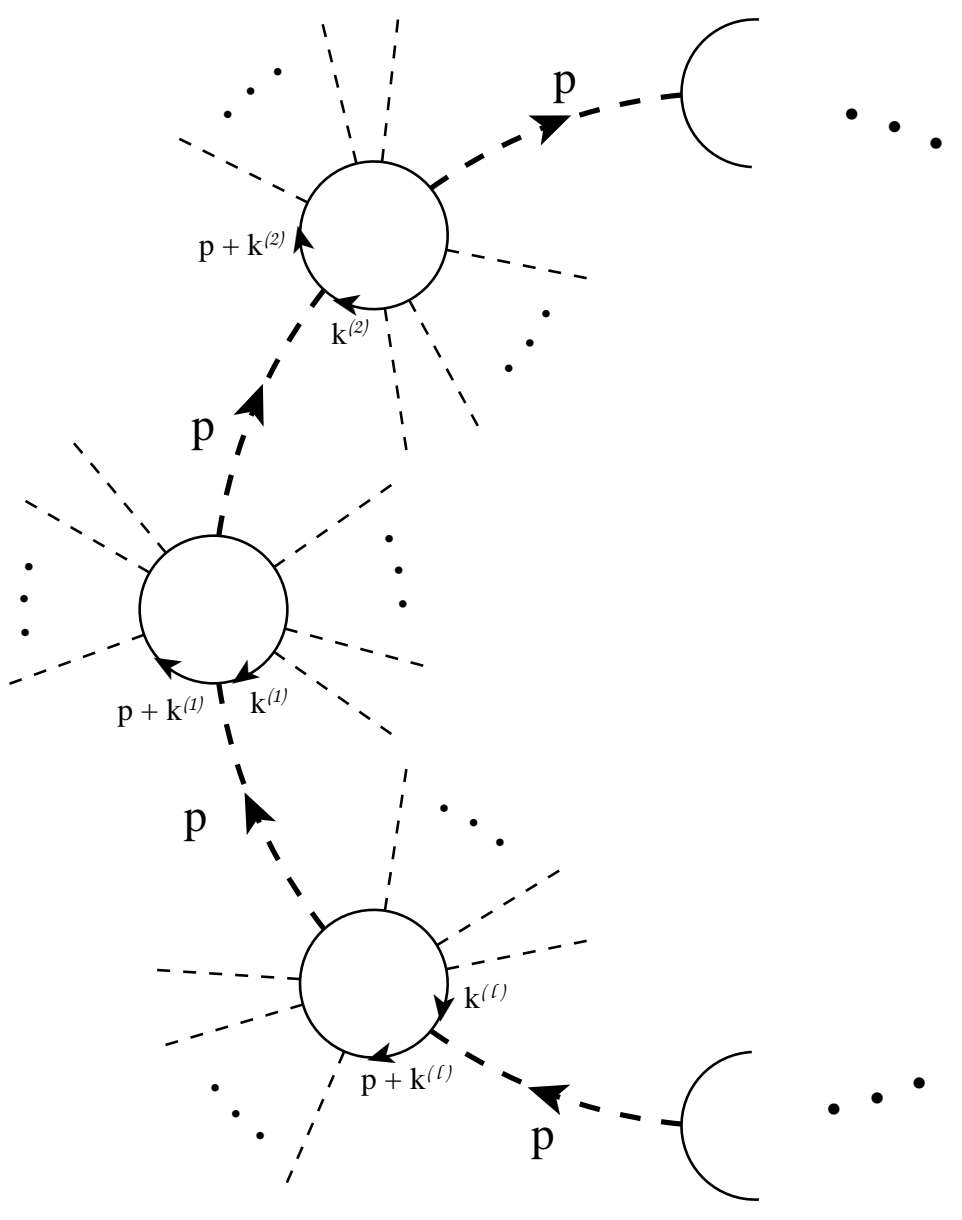

Fig. 2

Fig. 2: The $(\ell+1)$-loop 1-PI diagrams which contribute to the 1-PI Green functions which in turn yield the NTL part $V^{(1)}$ (beyond one loop) in $1 / N_{\mathrm{c}}$ expansion of $V_{\text {eff }}$. The diagrams contain $\ell$ loops of (massless) quarks. These loops are connected into another circle by $\ell$ propagators of the (nondynamical) scalars (all either Higgs, or neutral Goldstone, or charged Goldstones). In the case of charged Goldstone propagators, the quark loops are made up of the top and the bottom quark. 


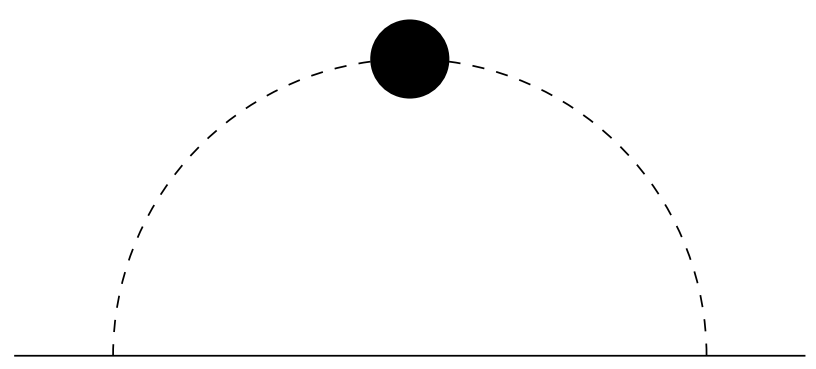

Fig. 3a

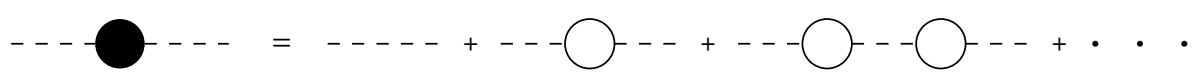

Fig. 3b

Fig. 3: the 1-PI diagrams with two external top quark legs which give the leading $\left(\mathcal{O}\left(1 / N_{\mathrm{C}}\right)\right)$ contribution to the renormalization of the mass $m_{t}$. Unlike the diagrams of Figs. 1-2, the top quark propagators here contain the nonzero bare mass $m_{t}(\Lambda)$ which was the solution to the NTL gap equation. The dashed lines are the propagators of the nondynamical scalars (either the Higgs, or the neutral Goldstones, or the charged Goldstones), while the dashed lines with a black blob are the propagators of the corresponding scalars that became dynamical through the NTL quantum effects. For the case of charged Goldstone propagators, the fermion loops contain one massive top quark and one massless bottom quark. 